\title{
Design of the future circular hadron collider beam vacuum chamber
}

\author{
I. Bellafont, ${ }^{1,2}$ M. Morrone $\odot,{ }^{2}$ L. Mether $\odot,{ }^{3,2}$ J. Fernández, ${ }^{4,2}$ R. Kersevan, ${ }^{2}$ \\ C. Garion, ${ }^{2}$ V. Baglin $\odot,{ }^{2}$ P. Chiggiato, ${ }^{2}$ and F. Pérez ${ }^{1}$ \\ ${ }^{1}$ ALBA Synchrotron Light Source, 08290 Cerdanyola del Vallès, Spain \\ ${ }^{2}$ CERN, The European Organization for Nuclear Research, CH-1211 Geneva, Switzerland \\ ${ }^{3}$ EPFL, Ecole Polytechnique Fédérale de Lausanne, CH-1015 Lausanne, Switzerland \\ ${ }^{4}$ CIEMAT, 28040 Madrid, Spain
}

(Received 15 October 2019; accepted 18 February 2020; published 6 March 2020)

\begin{abstract}
EuroCirCol is a conceptual design study of a post-LHC, Future Circular Hadron Collider (FCC-hh) which aims to expand the current energy and luminosity frontiers. The vacuum chamber of this $100 \mathrm{TeV}$, $100 \mathrm{~km}$ collider, will have to cope with unprecedented levels of synchrotron radiation linear power for proton colliders, 160 times higher than in the LHC for baseline parameters, releasing consequently much larger amounts of gas into the system. At the same time, it will be dealing with a tighter magnet aperture. In order to reach a good vacuum level, it has been necessary to find solutions beyond the particle colliders' state of art. This paper proposes a design of a novel beam screen, the element responsible for absorbing the emitted power. It is intended to overcome the drawbacks derived from the stronger synchrotron radiation while allowing at the same time a good beam quality.
\end{abstract}

DOI: 10.1103/PhysRevAccelBeams.23.033201

\section{INTRODUCTION}

The Future Circular Hadron Collider (FCC-hh) is a study aiming to propose a $100 \mathrm{~km}$ long accelerator as a successor of the $27 \mathrm{~km}$ long Large Hadron Collider (LHC) [1,2].

In the FCC-hh two counterrotating proton beams would achieve an energy of $50 \mathrm{TeV}$, leading to collisions at $100 \mathrm{TeV}$ at center of mass. Such energies require superconducting bending magnets providing up to $16 \mathrm{~T}$, an ambitious step forward with respect to the current $8.3 \mathrm{~T}$ of the LHC dipole magnets, which are needed to steer a $7 \mathrm{TeV}$ beam. This rise in beam energy results in a dramatic increase of the emitted synchrotron radiation (SR), attaining linear power density levels of $35.4 \mathrm{~W} / \mathrm{m}$, around 160 times higher than in the LHC, with a maximum of $0.22 \mathrm{~W} / \mathrm{m}$ (see Table I).

As in the LHC, the proposed FCC-hh's magnets are based on a two-in-one design, where the two beam pipes are incorporated into a common yoke cooled by superfluid $\mathrm{He}$ at $1.9 \mathrm{~K}$. Superfluid He allows an easier and more effective cooling of the magnet. In addition, at such low temperatures all gas species condense on a surface with saturated vapor pressures lower than $10^{-12}$ mbar, except for He.

To avoid excessive beam-induced heat load transfer to the $1.9 \mathrm{~K}$ surfaces, beam screens are inserted in the

Published by the American Physical Society under the terms of the Creative Commons Attribution 4.0 International license. Further distribution of this work must maintain attribution to the author(s) and the published article's title, journal citation, and DOI. magnet cold bores, aiming to intercept the SR power at higher temperatures. In this way, the cooling efficiency is increased [4].

This paper proposes a novel beam screen (BS) design for the FCC-hh, intended to meet with the requirements of such a challenging collider while coping with the detrimental effects arisen from the unprecedentedly high beam energy. The main challenges the FCC-hh BS has to overcome are: (i) the need of a higher pumping speed, to counter the higher gas load in the chamber (derived from the much higher SR power emission), (ii) the higher photoelectron generation (also derived from the higher SR), which may lead to an electron cloud ( $\mathrm{e}^{-}$cloud) build-up, (iii) the strong Lorentz forces generated during a magnet quench, derived from the huge dipole magnetic field, (iv) and the heat management.

These topics and the solutions adopted to address them are covered in this paper, paying special attention to the SR generation. The study of the gas generation and the vacuum level in the beam chamber is covered in another publication [5], owing to the otherwise unaffordable increase of length and complexity of the resulting paper.

\section{VACUUM SPECIFICATIONS}

Ultrahigh vacuum (UHV) is generally needed in particle accelerators to reduce beam-gas interaction at the required level. For a vacuum system, to quantify the residual gas remaining in the beam pipe, the gas density is reported instead of pressure when the vacuum vessels are at different temperature. This is the typical case of a set of vacuum chambers kept either at cryogenic or room temperatures. 
TABLE I. Comparison of the LHC's and the FCC-hh's relevant baseline parameters [2,3].

\begin{tabular}{lcc}
\hline \hline & LHC & FCC-hh \\
\hline Energy [TeV] & 7 & 50 \\
Current [mA] & 580 & 500 \\
Circumference [km] & 26.7 & 97.75 \\
Dipole max magnetic field [T] & 8.33 & 15.96 \\
Photon flux [ph/(m s)] & $1 \times 10^{17}$ & $1.7 \times 10^{17}$ \\
SR power [W/m arc dipole trajectory] & 0.22 & 35.4 \\
SR critical energy [eV] & 43.8 & 4286.3 \\
Cold bore aperture [mm] & 50 & 44 \\
Normalized emittance at 25 ns [ $\mu \mathrm{m}]$ & 3.75 & 2.2 \\
Angle between dipoles [ ${ }^{\circ}$ ] & 0.29 & 0.077 \\
Beam screen temperature range $[\mathrm{K}]$ & $5-20$ & $40-60$ \\
\hline \hline
\end{tabular}

As in the LHC, the BS of the FCC-hh is holed so that gas molecules can migrate to the coldest surface of the cold bore and be cryopumped, providing enough pumping speed to keep the gas density in the beam chamber below the maximum attainable one. The maximum gas density is defined by two constraints: (i) The nuclear scattering beam lifetime $\left(\tau_{b q}\right)$ has to be longer than $100 \mathrm{~h}$. (ii) The thermal load on the cold mass of the magnets (composed by all the elements held at $1.9 \mathrm{~K}$ that are directly cooled by the cryogenic system, as the coils, collars, iron yoke or the cold bore) attributed to nuclear scattering $\left(P_{n}\right)$ has to be lower than $0.2 \mathrm{~W} / \mathrm{m}$ in average. [6].

These constraints can be expressed with Eqs. (1) and (2). The gas density specification that fulfills both expressions (approximated by default) is the same as that of the LHC, [3,7], i.e., less than $1 \times 10^{15} \mathrm{H}_{2 \text { eq }} / \mathrm{m}^{3}$. $\mathrm{H}_{2 \text { eq }}$ means the equivalent pure $\mathrm{H}_{2}$ density once all the different nuclear scattering cross sections for other gas species have been taken into account. For this value, $\tau_{b g}$ results in $107.2 \mathrm{~h}$ and $P_{n}$ in $0.178 \mathrm{~W} / \mathrm{m}$.

$$
\begin{gathered}
\tau_{b g}=\frac{1}{\sigma c n}>100 h \\
P_{n}=k_{a}\left(1-k_{b}\right) \frac{I E}{c \tau_{b g}}<0.2 \mathrm{~W} / \mathrm{m}
\end{gathered}
$$

$\sigma$ is the nuclear scattering cross section, $86.4 \mathrm{mb}$ (taken from FLUKA [8,9]), $E$ the beam energy (in $\mathrm{eV}$ ), $I$ the beam current, $n$ the gas density; and $k_{a}$ is the fraction of the total scattered power in the arcs absorbed by the cold mass. For the latest design of the FCC-hh's vacuum chamber, $k_{a}$ has been found to be $\approx 0.86$ as an average in the arc cell [10]. The fraction of power deposited in the BS is only 0.05 . The remaining power is deposited in the tunnel walls or escapes. $k_{b}$ is defined as the fraction of protons whose interactions with the residual gas do not result in any energy deposition in the accelerator elements and continue around the ring, i.e., $\approx 0.042$ in the FCC-hh [11].

\section{SYNCHROTRON RADIATION IN THE FCC-hh}

Even if designed for a slightly lower beam currents than the LHC, the high beam energy of the FCC-hh results in a dramatic increase of the SR power $(P)$ and critical energy $\left(\varepsilon_{c}\right)$. To allow a rapid comparison, these two terms are plotted for both colliders in Figs. 1 and 2 as a function of the beam energy, using Eqs. (4) and 2. They have been derived from the expressions found in $[12,13]$.

$$
\begin{gathered}
\dot{\Gamma}[\mathrm{ph} /(\mathrm{m} \mathrm{s})]=7.007 \times 10^{16} \frac{E[\mathrm{TeV}]}{\rho[\mathrm{m}]} I[\mathrm{~mA}] \\
P[\mathrm{~W} / \mathrm{m}]=1.239 \frac{E^{4}[\mathrm{TeV}]}{\rho^{2}[\mathrm{~m}]} I[\mathrm{~mA}] \\
\varepsilon_{c}[\mathrm{eV}]=3.583 \times 10^{2} \frac{E^{3}[\mathrm{TeV}]}{\rho[\mathrm{m}]}
\end{gathered}
$$

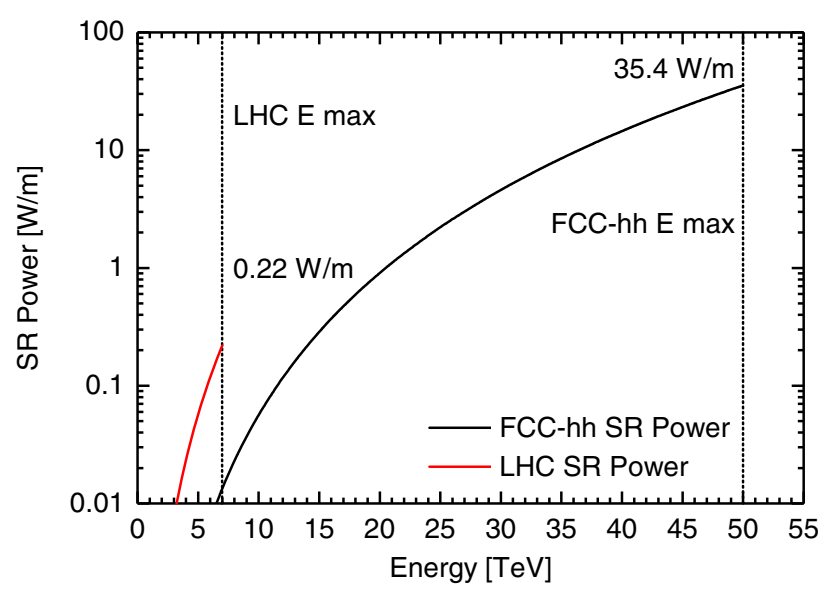

FIG. 1. Comparison of SR power generated per arc dipole trajectory vs beam energy, for the FCC-hh and the LHC. $500 \mathrm{~mA}$.

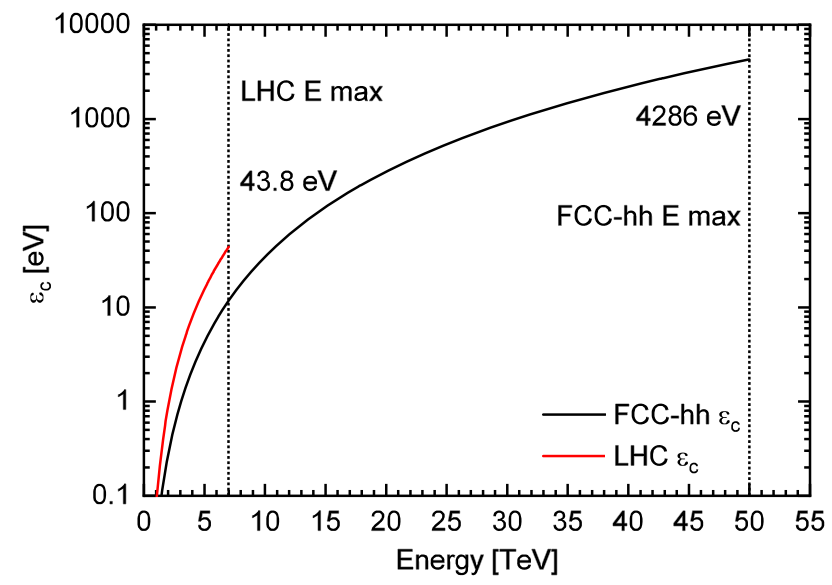

FIG. 2. Comparison of $\varepsilon_{c}$ vs beam energy, for the FCC-hh and the LHC. 


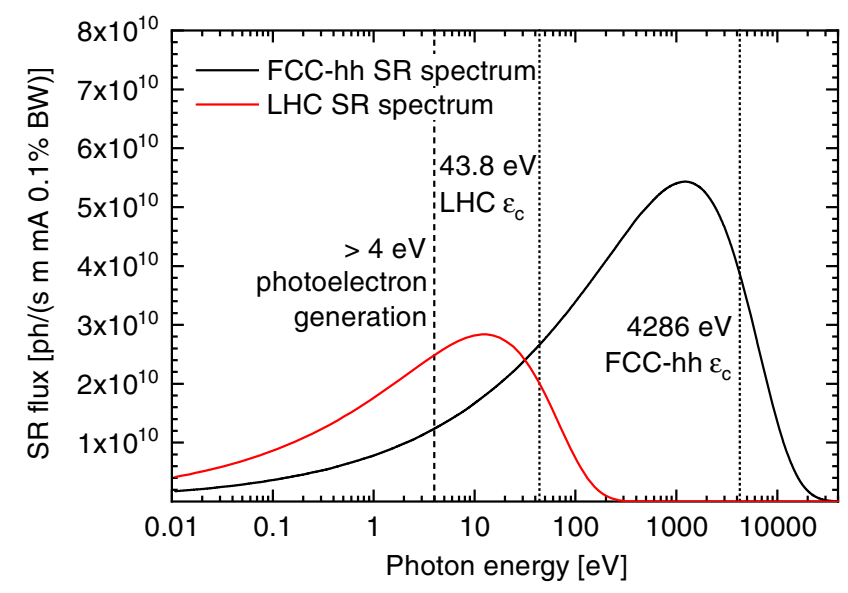

FIG. 3. Comparison of the FCC-hh's SR spectrum vs the LHC's. BW = Band Width.

Compared to the LHC, the linear SR power density in the FCC-hh is $\approx 160$ times higher, namely of the order of magnitude of modern synchrotron radiation sources. However, in the range of energies of the LHC (0.45$7 \mathrm{TeV}$ ), both the FCC-hh's SR $P$ and $\varepsilon_{c}$ are lower, due to the larger radius of magnetic curvature $(\rho)$, around $10.45 \mathrm{~km}$ in the FCC and $2.8 \mathrm{~km}$ in the LHC.

A comparison of the SR spectrum generated by the two colliders can be found in Fig. 3. At maximum beam energy, most of the photon flux in the LHC is generated in the infrared-UV region $\left(1.24 \times 10^{-3}-100 \mathrm{eV}\right.$, around 95\%), and a marginal part in the soft $\mathrm{x}$-ray region, $(>100 \mathrm{eV}$, with only around $2 \%$ of the total emitted flux). In FCC-hh around $66 \%$ of the photons are emitted in the soft and hard $\mathrm{x}$-ray region.

One of the hypotheses present in the literature which explains the photon stimulated desorption (PSD), describes a mechanism in which photoelectrons are the source of the gas generation [14]. The extraction of photoelectrons from the chamber wall needs photon energy higher than $4-5 \mathrm{eV}$, i.e., the work functions of metals usually employed in UHV. Therefore, photons below this energy will not contribute substantially to the increase of the gas density inside of the vacuum chamber. In the LHC, for design parameters, the photon flux is $1 \times 10^{17} \mathrm{ph} /(\mathrm{ms})$ [see Eq. (3)], with $48 \%$ of this amount above $4 \mathrm{eV}$. In the FCC-hh, the photon flux is $1.7 \times 10^{17} \mathrm{ph} /(\mathrm{m} \mathrm{s})$ with $88 \%$ of the photon energies above $4 \mathrm{eV}$. On the assumption that photoelectrons are the source of PSD, this would mean that in the FCC-hh there are around 3 times more photons emitted per meter capable of increasing the gas load in the beam chamber.

\section{THE BEAM SCREEN}

The BS serves several purposes [15]. Among them, the most relevant one is the reduction of the SR power arriving to the cold bore [3], by directly absorbing it at higher temperatures. The removal of $1 \mathrm{~W}$ at $1.9 \mathrm{~K}$ requires nearly $1 \mathrm{~kW}$ of electric power, which would be translated in around 2.3 GW of cooling power for all the FCC-hh in case of the BS absence, making the machine totally unfeasible. From the vacuum point of view, its most important function is to screen of the gas condensed on the cold bore from the SR direct impact, avoiding the desorption of the accumulated gas back into the system [16] and the consequent drastic reduction of pumping speed. In addition, the BS is also responsible of mitigating the $\mathrm{e}^{-}$cloud effect generated by the beam's presence and of ensuring a sufficiently low beam impedance. At the same time, the BS must preserve the magnetic field quality and the minimum clearance for the beam, has to respect the tight aperture of the magnet bore, and has to ensure its structural integrity during the magnet quenches.

The latest FCC-hh BS design for dipole magnets is shown in Fig. 4. The BS elements and their main purpose are hereunder presented.

\section{A. Primary chamber}

The primary or inner chamber is the innermost part of the beam screen. Its volume is delimited by two $1.3 \mathrm{~mm}$ thick copper colaminated P506 [17] stainless steel (SS) sheets. The P506 SS, $1 \mathrm{~mm}$ thick, is used to achieve a high stiffness while yielding low relative magnetic permeability $(<1.005)$. The OFE copper layer is $0.3 \mathrm{~mm}$ thick (in the LHC it was $0.075 \mathrm{~mm}$ thick [18]) and has a RRR of at least 100. It is used to achieve low impedance values. Based on machine optics considerations, the inner chamber has to guarantee a clearance to contain a $15.5 \sigma$ beam aperture [19] while yielding a low beam impedance. The inner copper surface is kept as cold as possible to minimize the copper's resistivity [20]. Provided that the $\mathrm{e}^{-}$cloud effect is effectively suppressed, the primary chamber does not receive any significant heat load besides of that of the image currents The SR reflected back from the secondary chamber is minimal, and only the outer angular extremes of the SR beam hit it directly, carrying a negligible amount of power. Its temperature is thus directly determined by that of the BS coolant (supercritical helium), with less than $0.5 \mathrm{~K}$ of difference.

The central slot in the inner chamber, which leads to the secondary one, has an aperture of $7.5 \mathrm{~mm}$. It is optimized to transfer $99.9 \%$ of the generated SR power to the secondary chamber even for the worst case of $2 \mathrm{~mm}$ vertical misalignment (see Fig. 5), whilst covering the inner area of the secondary chamber as much as possible.

During the beam injection at $3.3 \mathrm{TeV}$, the vertical misalignment could go up to $4 \mathrm{~mm}$ for a short time [21], during which the SR beam would hit directly the wall of the primary chamber. Nevertheless for that low beam energy the SR $P$ and $\varepsilon_{c}$ are considerably low, resulting in negligible temperature variation and gas desorption rate. 


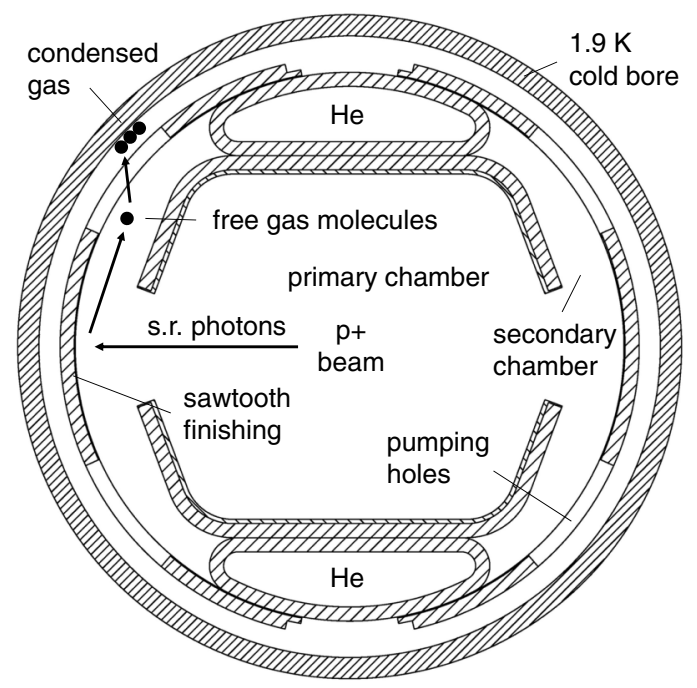

(a) Cross section of the FCC-hh beam screen inside the cold bore

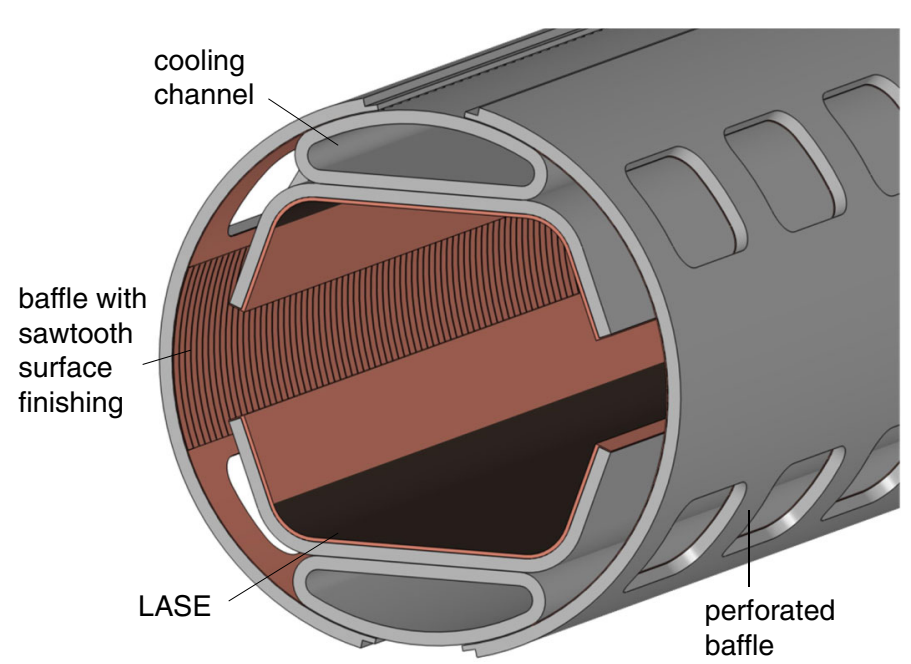

(b) View of the FCC-hh beam screen with the sawtooth surface finishing applied on the irradiated baffle

FIG. 4. FCC-hh beam screen for bending magnets, featuring LASE treatment on the upper and lower flat areas of the inner chamber.

The edges of the colaminated P506 SS inner chamber sheets, which mark the boundaries of the $7.5 \mathrm{~mm}$ slot, are coated with $100 \mu \mathrm{m}$ of copper to keep the impedance within the requirements. Even if they would present a very small SS surface exposed to the beam's sight in case of not being coated, SS is three orders of magnitude more resistive than copper at cryogenic temperatures, surpassing the allocated impedance budget and being necessary to cover it. The chosen coating solution has to guarantee an electrical conductivity of at least $6.5 \times 10^{8} \mathrm{~S} / \mathrm{m}$ at $50 \mathrm{~K}$ [22]. Cold spray and electrodeposition are the initially envisaged options, which are compatible with the thermomechanical behavior of the BS. Additional studies are required to fully assess the different technological options and features to produce this copper layer on the edge in a reliable and cost effective way.

To mitigate the $\mathrm{e}^{-}$cloud effect, it is proposed to treat part of the inner chamber surface with Laser Ablation Surface Engineering (LASE) [23-26] or to coat it with amorphous carbon $(\mathrm{a}-\mathrm{C})[27,28]$. These treatments are able to lower the secondary electron yield (SEY) below 1 for a range of electron energy of $0-1000 \mathrm{eV}$. From the manufacturing point of view, LASE is preferred over a-C since it is possible to apply it during the series production under atmospheric pressure, lowering considerably the manufacturing costs if scaled up to the $100 \mathrm{~km}$ twin-bore machine. The drawback that LASE entails, however, is a worse surface resistance owing to its high aspect ratio. That being said, its resistance can be minimized if the ablation ratio, and thus the SEY reduction, are low [29]; and/or if the treatment is applied in parallel to the beam's direction, achieving at cryogenic temperatures surface resistance values quite similar to a-C ones, even for high ablation rates [30].

\section{B. Secondary chambers}

Two lateral baffles, which are symmetrically assembled, close horizontally the annular space between the primary and secondary chambers. These baffles are composed of $1 \mathrm{~mm}$ thick P506 SS sheet and a $75 \mu \mathrm{m}$ copper layer, which acts as a heat carrier. The thickness of this layer, same value as in the LHC's BS, has been optimized in order to minimize at the same time the forces generated during a magnet quench and the temperature increase on the irradiated baffle (less copper means less force but also less heat transfer). The SR fan hits directly one of the baffles of the secondary chambers with $\approx 29 \mathrm{~W} / \mathrm{m}$ in average and with an approximated vertical size of $2 \mathrm{~mm}$ (see Fig. 5). The average grazing angle of incidence of the

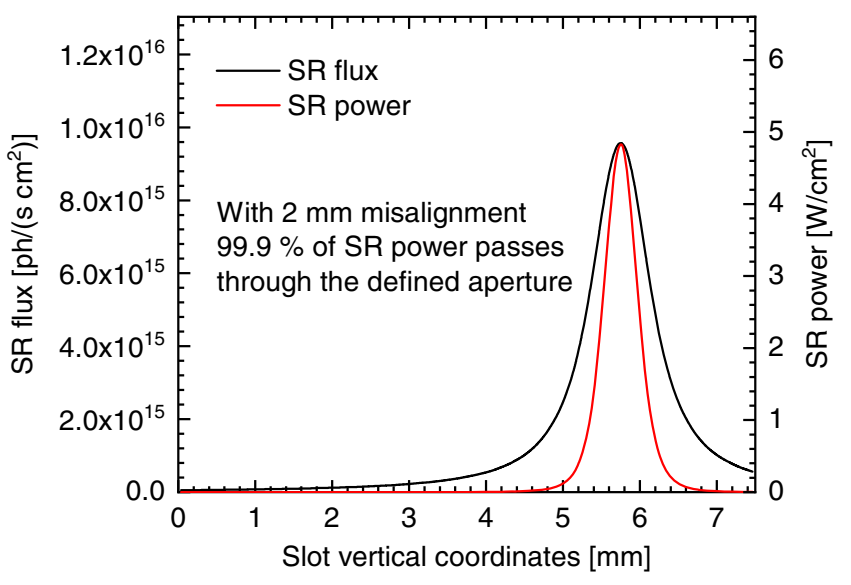

FIG. 5. SR flux and power baseline distribution, passing through the $7.5 \mathrm{~mm}$ slot of the primary chamber up to the secondary one. Case of maximum contemplated vertical misalignment. 
SR on the BS is $0.10^{\circ}(1.8 \mathrm{mrad})$, higher than the angular offset, $0.077^{\circ}$, due to the long travel path of the photons, which causes that the SR emitted at the end of each bending magnet (MB) misses the following magnet and impacts on the 2nd one in the line, with doubled angular offset.

A sawtooth surface finishing is present on the irradiated baffle, as in the LHC. This finishing, applied by means of a roller with a jagged relief on its surface, leaves perpendicular triangular teeth in the SR trajectory, setting the grazing incidence angle close to $90^{\circ}$. Given the hardness of P506 SS, the copper layer is also needed for this reason. Owing to the reflectivity properties of $\mathrm{x}$-rays, the perpendicular incidence increases the SR absorption on the impact area. Being the average impact grazing angle in the FCC-hh much lower than in the LHC, the LHC's sawtooth structure has been adapted, making the teeth two times longer. This minimizes the amount of SR hitting the rounded tips of the teeth (present due to manufacturing limits), which increase the residual SR scattering. In order to properly model these rounded areas in the computer tools, an LHC BS sample was measured with an optical profilometer. Results are shown in Fig. 6. A dedicated experimental plan led by LNF-INFN (Frascati, Italy) was also arranged with the objective of measuring the reflectivity and photoelectron yield of the sawtooth surface and other materials used for the beam screen, in the optics beamline of BESSY-II light source [31]. With the obtained data [32-34], the simulations were improved and validated, and an equivalent and simple model of the sawtooth surface was created in order to save computing resources. As a conservative approach, the area of the found surface has been multiplied by a standard factor of two, enhancing the resulting reflectivity.

Figure 7 displays the results of the simulated reflectivity of an ideal sawtooth surface compared with a nonideal, pessimistic one, without perfectly sharp teeth. The theoretical reflectivity of an untreated copper surface is also shown. It can be noticed how this treatment is highly efficient in absorbing high energy photons. For the proposed sawtooth profile, the performed simulations show an

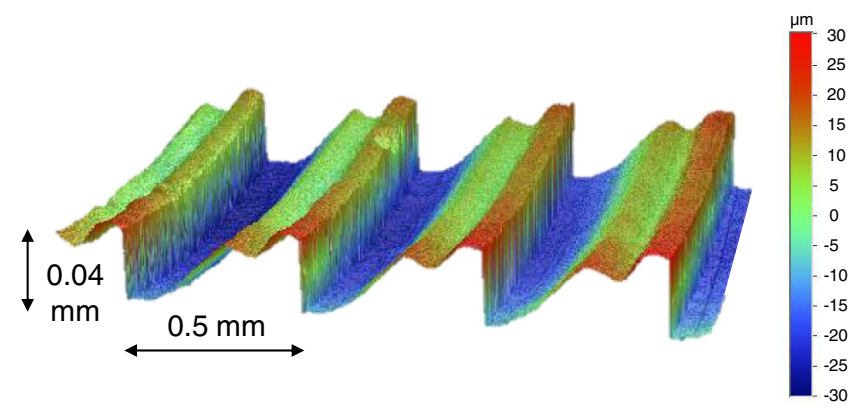

FIG. 6. Close-up view of the surface of an LHC BS sawtooth, with $40 \mu \mathrm{m}$ of average height per tooth and $500 \mu \mathrm{m}$ of pitch. Measured at CERN. absorption of around $98 \%$ of the total incident SR power and more than the $80 \%$ of the total photon flux at $50 \mathrm{TeV}$.

If no sawtooth finishing was present, the absorption would be around $46 \%$ for the power and $20 \%$ for the flux. With this surface finishing the gas load attributed to PSD is lowered, since the total irradiated area is smaller and the SR incidence perpendicular. The number of photons reflected back to the primary chamber is also diminished, lowering the generation rate of $\mathrm{e}^{-}$seeds for the $\mathrm{e}^{-}$cloud effect $\left(N_{e}\right)$. In case that an improvement of the SR absorption was required, there is the possibility of increasing the roughness of the rounded areas treating them with LASE, with the initially envisaged drawbacks of increasing the manufacturing costs and surface resistance. Thanks to its high surface aspect ratio, LASE provides an exceptionally high absorption rate, as found in the performed experiments [32-34]. Furthermore, using LASE on the sawtooth would also result in a further reduction of the gas load due to its low PSD molecular desorption yield [36], and due to the lower $N_{e}$ in the inner chamber (see Sec. V). It is encouraged to study this strategy in the future.

In order to allow the gas to reach the cold bore, each baffle has two rows of pumping holes designed to maximize the pumping speed while minimizing the SR leaked to the $1.9 \mathrm{~K}$ cold bore and guaranteeing enough mechanical stiffness. The pumping holes are placed behind the inner chamber, as far as possible from the SR impact area [see Fig. 4(a)], being protected from a direct irradiation by the SR and from the $\mathrm{e}^{-}$cloud impingement, since electrons generated on the sawtooth surface are forced to follow the magnetic field lines (see Fig. 11), and the baffle's curvature prevents a vertical leakage. Electrons generated close to the pumping holes, do not receive any significant kick from the beam's positive space/charge, preventing their multiplication in the secondary chamber. Thanks to this double

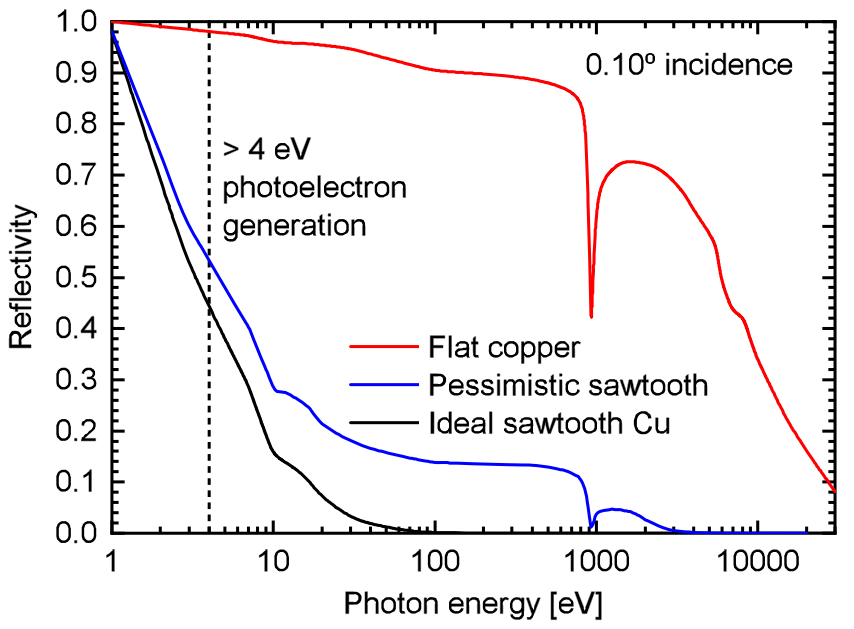

FIG. 7. Total reflectivity comparison of a flat copper surface, with $\tau=0.006$ and for the FCC-hh's average SR impact grazing angle, vs the same surface with an ideal sawtooth finishing and vs nonideal, pessimistic one. Data obtained with SYNRAD+ [35]. 
chamber layout, the electron shields present in the LHC's BS [37] are no longer necessary. In addition, since the beam has no direct sight of the pumping holes, their contribution to the BS impedance is negligible. Not being the impedance a constraint, and being protected from the direct SR irradiation, the pumping holes can be much larger than in the LHC, enhancing in this way the pumping speed. Without the double chamber layout, their dimensions would be unaffordable [38].

\section{Cooling channels}

Two P506 SS cooling channels are placed top and bottom of the BS. They are welded to the inner chamber sheets and to the lateral baffles. Supercritical He flows through them, cooling half a cell in a row $(\approx 107 \mathrm{~m})$. At nominal current and beam energy, the $\mathrm{He}$ is at $40 \mathrm{~K}$ in the inlet and $57 \mathrm{~K}$ at the outlet [39]. Compared with the LHC cooling channels, a considerable increase of the cross section area was necessary to dissipate the higher SR power $(35.4 \mathrm{~W} / \mathrm{m}$ vs $0.22 \mathrm{~W} / \mathrm{m})$, reaching a heat transfer coefficient of $5000 \mathrm{~W} /\left(\mathrm{K} \mathrm{m}^{2}\right)$ [40] and 50 bar of pressure.

\section{Cold bore}

The cold bore is a SS $316 \mathrm{LN}$ pipe, $1.5 \mathrm{~mm}$ thick and with an inner diameter of $44 \mathrm{~mm}$. It is kept as $1.9 \mathrm{~K}$ and it is the only means of pumping in the machine during normal operation. It separates the superfluid He surrounding the magnet coils and the vacuum chamber. The BS is supported inside the cold bore by means of periodic P506 SS spring sets every $750 \mathrm{~mm}$, designed to minimize the heat conduction to the cold bore and ease the insertion of the BS inside it (see Figs. 20 and 23). The solution used in the LHC, short bi-metallic rings [41,42], has been discarded for the FCC-hh. Even if cheaper, they are not so efficient at isolating thermally the BS from the cold bore, an effect which would be exacerbated in this new BS due to its higher temperature.

\section{E. Interconnects}

The continuity of the beam screen in the arcs is broken by the magnets interconnects, in which the SS bellows and RF fingers absorb the offset angle between the magnets, thermal displacements and mechanical tolerances. In order to protect these areas of direct irradiation, a copper absorber, shown in Fig. 8, is proposed to be placed at the end of each magnet, stopping a maximum of $41 \mathrm{~W}$ of SR power and delivering a shadow of around $1.2 \mathrm{~m}$ afterwards, following the beam direction. The absorber slope should be treated with LASE to minimize the SR scattering and photoelectron generation, as it is difficult to apply a sawtooth finishing to this area. As shown in Table IV, the resulting power in the copper transition pieces, rf fingers and bellows of the interconnect is less than $0.2 \mathrm{~W}$, effectively excluding this area of requiring

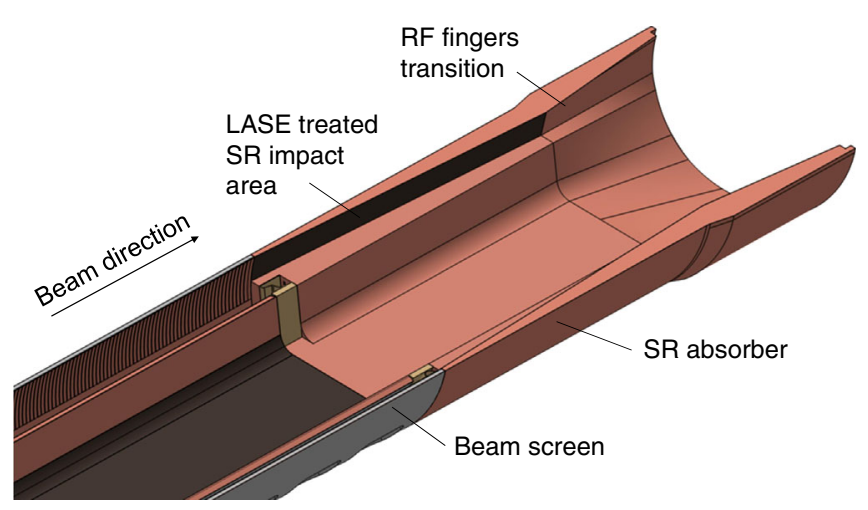

FIG. 8. FCC-hh photon absorber conceptual representation.

active cooling and minimizing the related outgassing. Other solutions to avoid the irradiation of the rf fingers are also feasible. The absorber can be shortened or even removed, as long as the diameter of the rf fingers and its adjacent transition elements is larger than the BS, absorbing a very small amount of SR on the last copper transition, which should also include LASE.

\section{F. General remarks}

The presented BS is intended to minimize as much as possible the beam impedance. The impedance calculation, however, has been proven to be challenging due to lack of maturity in the studies carried out on LASE technology. The resulting pumping speed is considerably high, surpassing the LHC's even at the same normalized temperature, and being sufficient to guarantee the gas density requirement within a reasonable conditioning time [5]. The calculated values are shown in Table II. The calculation done with the outgassing $(Q)$ applied on the sawtooth, represents the closest case to the reality, where PSD dominates the gas load. When $Q$ is applied on the inner chamber, it represents a pessimistic calculation, where all the gas desorption happens on the inner copper layer (caused either by electrons or reflected photons) being this value the lowest attainable. Even if the complexity of the FCC-hh BS is much higher than that of the LHC, it is also compatible with large scale production technologies [43] and affordable from the economic point of view, representing a very small fraction of the collider's cost [44].

TABLE II. Comparison of the LHC's and the FCC-hh's pumping speeds for $\mathrm{H}_{2}$. Calculated at the beam's path, with MOLFLOW+ $[35,45,46]$, for an infinite pipe and different outgas$\operatorname{sing}(Q)$ sources.

\begin{tabular}{lcc}
\hline \hline & LHC & FCC-hh \\
\hline Temperature window $[\mathrm{K}]$ & $5-20$ & $40-60$ \\
Nominal $(5 / 40 \mathrm{~K}) . Q$ on inner ch. $[1 /(\mathrm{s} \mathrm{m})]$ & 173 & 898 \\
40 K. $Q$ applied on inner chamber $[1 /(\mathrm{s} \mathrm{m})]$ & 489 & 898 \\
40 K. $Q$ applied on sawtooth $[1 /(\mathrm{s} \mathrm{m})]$ & 493 & 1125 \\
\hline \hline
\end{tabular}




\section{V. $\mathrm{E}^{-}$CLOUD MITIGATION}

The secondary electron emission of the vacuum chamber surfaces can drive an avalanche multiplication effect, filling the beam chamber with a cloud of electrons. The interaction of the proton beam and the $\mathrm{e}^{-}$cloud can lead to a series of detrimental effects on the collider's performance, such as emittance growth, transverse instabilities, heat load on the surfaces bombarded by electrons, and a deterioration of the vacuum quality owing to the electron stimulated desorption. The BS has to therefore comply to a series of design constraints in order to achieve a low electron density and minimize its impact on the collider's performance.

The $\mathrm{e}^{-}$cloud build up depends on the SEY of the chamber surfaces, on the chamber geometry, the beam current, the bunch spacing, and the photoelectron generation rate. Within the parameters depending on the BS design, the SEY features the highest influence on the electron density. A series of SEY constraints have been therefore defined. As a first step, these requirements are expressed in a fast way through the multipacting threshold, namely the maximum value of the SEY curve above which the exponential electron multiplication starts independently from the number of photoelectron seeds. They have been estimated with simulation studies of $\mathrm{e}^{-}$cloud build-up with the PyECLOUD code $[47,48]$, using a secondary emission model [49-51] based on measurements on samples of the LHC copper co-laminated beam screens [52-54]. The calculated SEY requirements can be found in Table III. The requirements have been calculated for each bunch spacing option, for dipoles, quadrupoles and drift spaces (without magnetic field), and for nominal and injection energies. $12.5 \mathrm{~ns}$ results to be the most demanding option, whilst $25 \mathrm{~ns}$, the FCC-hh's nominal value, is the least demanding one.

Conditioned copper can reach SEY values of around 1.2-1.4, as displayed in Fig. 9. Consequently, it is necessary to use a SEY mitigation solution for all the quadrupole magnets, which require a lower SEY, at least 1.1 in the best scenario. For the dipole magnets, in case the $12.5 \mathrm{~ns}$ bunch spacing option were decided to be discarded, raw, untreated copper could be used if conditioned. Otherwise, since the $12.5 \mathrm{~ns}$ option requires an SEY < 1.1, a SEY mitigation solution should be applied on them. As for the drift spaces, the calculation is indicative, but they do not present any strong requirement.

For the common range of electron energies in the beam chamber, LASE can reach SEY values under the unity even without beam conditioning, and well below one after high doses (see Fig. 9). Nevertheless, it is relevant to point out that there are different properties and SEY values that LASE can present, depending among other factors on the surface ablation level [24], a feature which increases the surface aspect ratio and apparent blackness. The improvement in SEY is proportional to this feature, but it also affects the surface resistance [25]. Therefore, and as future

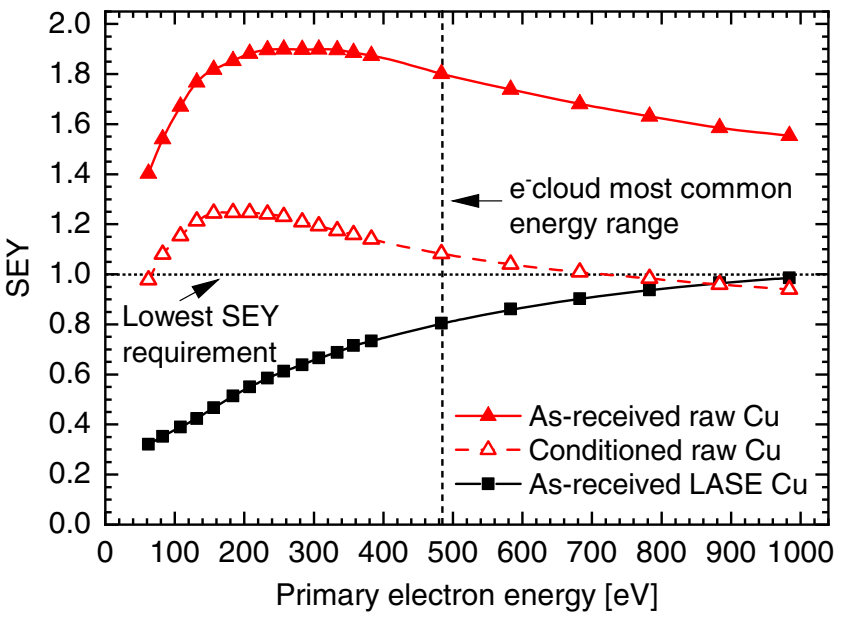

FIG. 9. Comparison of SEY measured on LASE [25] and on raw $\mathrm{Cu}$ [23] as taken from the literature. The expected range of most common electron energies $(0-485 \mathrm{eV}$, reading the average electron energy along the transverse plane of the dipole BS) is also shown, using $\mathrm{Cu}$-like SEY curves in the simulation.

work, and in case LASE is finally accepted as the chosen solution for the FCC-hh, it is important to adjust the properties of this treatment to match optimally the SEY requirements minimizing at the same time its impact on the impedance. More information of the FCC-hh's baseline LASE parameters can be found in [55].

Additionally, even if the SEY is below the requirements (see Table III), transverse instabilities can happen if the electron density in the chamber is high enough. The maximum allowable electron density $\left(\rho_{e, t h}\right)$ can be estimated using Eqs. (6)-(9) [56,57]:

$$
\rho_{e, t h}=\frac{2 \gamma \nu_{s} \omega_{e} \sigma_{z} / c}{\sqrt{3} K Q r_{p} \beta_{x, y} L}
$$

where $\omega_{e}, K$, and $Q$ are defined as:

$$
\begin{gathered}
\omega_{e}=\sqrt{\frac{\lambda_{p} r_{e} c^{2}}{\sigma_{y}\left(\sigma_{x}+\sigma_{y}\right)}} \\
K=\omega_{e} \sigma_{z} / c \\
Q=\min \left(\omega_{e} \sigma_{z} / c, 7\right)
\end{gathered}
$$

\begin{tabular}{|c|c|c|c|c|c|c|}
\hline \multirow{2}{*}{$\frac{\text { Bunch spacing }}{\text { Beam energy }[\mathrm{TeV}]}$} & \multicolumn{2}{|c|}{$25 \mathrm{~ns}$} & \multicolumn{2}{|c|}{$12.5 \mathrm{~ns}$} & \multicolumn{2}{|c|}{$5 \mathrm{~ns}$} \\
\hline & 3.3 & 50 & 3.3 & 50 & 3.3 & 50 \\
\hline Dipole & 1.5 & 1.5 & 1.1 & 1.1 & 1.5 & 1.5 \\
\hline Quadrupole & 1.1 & 1.2 & 1.0 & 1.0 & 1.1 & 1.0 \\
\hline Drift space & 2.0 & 2.0 & 1.3 & 1.3 & 1.6 & 1.6 \\
\hline
\end{tabular}

TABLE III. SEY requirements to avoid reaching the multipacting threshold. 
where $r_{p}$ and $r_{e}$ are the classical proton and electron radii, $\nu_{s}$ is the synchrotron tune, $\lambda_{p}$ is the bunch line density, $\sigma_{x, y, z}$ are the RMS transverse beam sizes and bunch length, $\beta_{x, y}$ are the machine beta functions and $L$ is the length of the machine over which the $\mathrm{e}^{-}$cloud extends. Using FCC-hh specifications, the threshold electron density results in $6 \times 10^{10} \mathrm{e}^{-} / \mathrm{m}^{3}$ at $3.3 \mathrm{TeV}$ (injection) and $3.6 \times 10^{11} \mathrm{e}^{-} / \mathrm{m}^{3}$ at $50 \mathrm{TeV}$ (physics).

For the SEY and electron generation rate $\left(N_{e}\right)$ values below the multipacting threshold, the electron density is approximately proportional $N_{e}$ on the areas where the $\mathrm{e}^{-}$ cloud occurs, as seen in Fig. 10. To prevent surpassing the threshold and keep the gas density low, it is advisable to keep $N_{e}$ in the inner chamber well below $1 \times 10^{12} \mathrm{e}^{-} /\left(\mathrm{cm}^{2} \mathrm{~s}\right) \cdot N_{e}$ depends on the SR flux arriving on the surface. It can be found with Eq. (10):

$$
N_{e}=\int_{E_{\min }}^{E_{\max }} \dot{\Gamma}_{p h}(E) Y_{p h}(E) d E
$$

where $E_{\max }$ and $E_{\min }$ are the maximum and minimum values of the SR spectrum arriving to the studied area, $\dot{\Gamma}_{p h}$ is the photon flux associated for each energy value and $Y_{p h}$ the photoelectron yield, the amount of electrons released by the surface for each impinging photon.

The $Y_{p h}$ for LHC copper and LASE was found in BESSY's experimental runs [32-34], for a range of photon energies of 35-1800 eV and angles between $0.25^{\circ}$ and $1^{\circ}$. $Y_{p h}$ values for $4-50 \mathrm{eV}$ were linearly extrapolated. The SR spectrum and flux arriving to the studied regions are given by the ray tracing simulations (see Sec. VI). Thanks to the sawtooth finishing, the energy of the reflected photons which reach the main chamber is very low, as shown in Fig. 15.

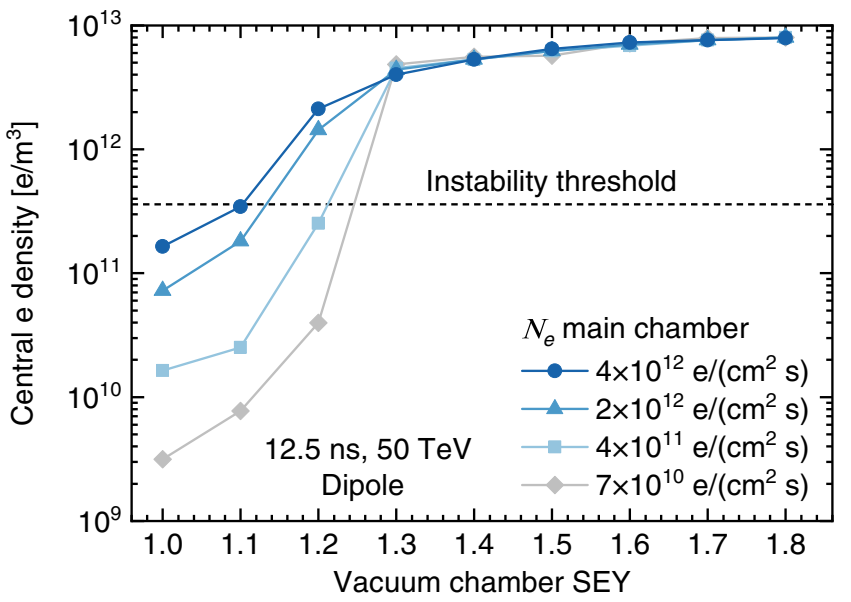

FIG. 10. Calculated electron density in the beam region depending on photoelectron generation rate and SEY, in a dipole chamber with $12.5 \mathrm{~ns} 50 \mathrm{TeV}$ beam.
The $N_{e}$ maximum calculated values during physics are $2.3 \times 10^{10} \mathrm{e}^{-} /\left(\mathrm{cm}^{2} \mathrm{~s}\right)$ for the dipole critical build-up areas (top and bottom flat areas in the primary chamber) and $1.6 \times 10^{11} \mathrm{e}^{-} /\left(\mathrm{cm}^{2} \mathrm{~s}\right)$ for quadrupole ones, if LASE is used. If using raw copper, the corresponding values are $1 \times 10^{11} \mathrm{e}^{-} /\left(\mathrm{cm}^{2} \mathrm{~s}\right)$ and $6 \times 10^{11} \mathrm{e}^{-} /\left(\mathrm{cm}^{2} \mathrm{~s}\right)$, respectively. In all cases $N_{e}$ has an associated electron density under the instability threshold (see Fig. 10 for an example of the dipole with the $12.5 \mathrm{~ns}$ beam, with the instability threshold drawn), mainly thanks to the high SR absorption properties of the sawtooth finishing, which lowers considerably the number of photons reaching the critical areas. During the beam's injection, the lower photon flux [15 times lower, see Eq. (3)] and lower $\varepsilon_{c}(1.23 \mathrm{eV}$, much lower than copper's work function) entail negligible $N_{e}$ values when compared with the physics mode, rendering the latter the only concern.

Treating the rounded areas of the sawtooth profile with LASE, and/or having sawtooth not only in the irradiated baffle but also in the other one will further lower the $\dot{\Gamma}_{p h}$ reflected toward the build-up areas and thus $N_{e}$, meaning in turn lower gas load ascribed to the electron stimulated desorption effect.

Figure 11 shows the BS electron density map in dipoles and quadrupoles for an early version of the BS. It can be seen how the electrons are confined around the magnetic field direction lines, impacting on the top and bottom flat areas of the BS in case of the dipoles and on the corners in case of quadrupoles. The production of photoelectrons in the secondary chamber is not contributing significantly to the density value around the beam, thanks to the magnetic confinement. In contrast to Fig. 4(b), where a LASE layout for dipoles is displayed, in the quadrupoles case LASE shall be applied only on the corners of the inner chamber, every $90^{\circ}$, where the $\mathrm{e}^{-}$cloud impacts. The use of LASE in the drift spaces between magnets, with a much lower magnetic field, is still under study.

A SEM image of the LASE sample whose reflectivity and $Y_{p h}$ were analysed is found in Fig. 12. The sample was provided by STFC (Science and Technology Facilities

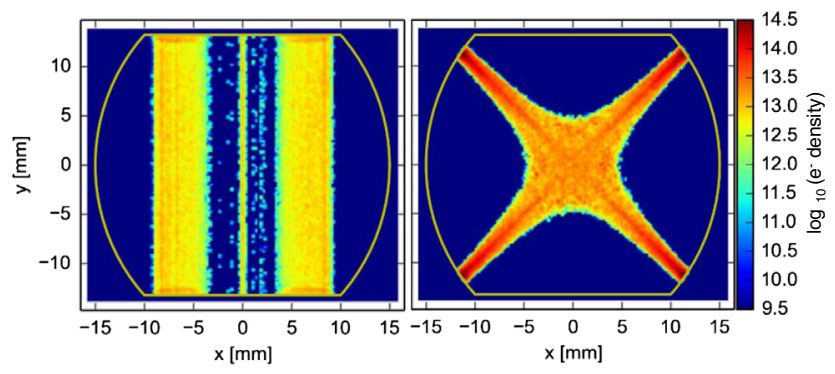

FIG. 11. Electron density distributions for an early version of the BS with the FCC-hh parameters for the $25 \mathrm{~ns}$ beam option. On the left, BS in a dipole magnetic field, on the right in a quadrupole magnetic field. 


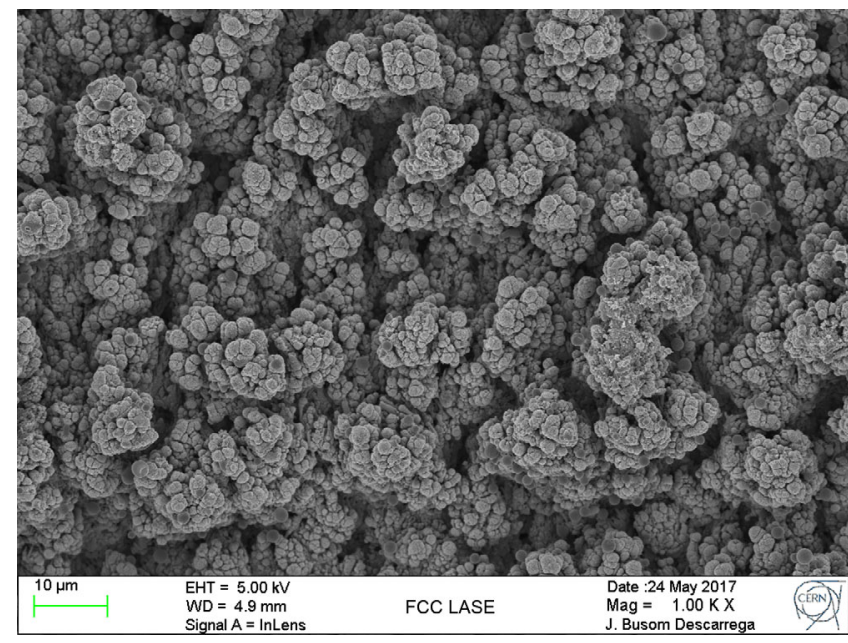

FIG. 12. 1.00 K SEM image of a $\mathrm{Cu}$ baseline LASE sample. The high roughness characteristic of this treatment can be easily observed. Measured at CERN.

Council, UK) according to the baseline specifications. The high aspect ratio the surface exhibits can be observed. This feature is thought to be the main reason for the SEY and $Y_{p h}$ reductions. The electrons become trapped inside the complex morphology, and the light incidence becomes perpendicular against the roughness peaks. $\mathrm{e}^{-}$cloud mitigation based on LASE has been recently demonstrated in an accelerator for the first time [58], with positive results.

\section{SYNCHROTRON RADIATION RAY TRACING}

In order to check the BS mechanical stability and to know the pressure levels in the vacuum chamber, several Monte Carlo and finite element simulations have been carried out. These studies need a complete map of both the photon flux and power absorbed along the vacuum chamber, found with photon ray tracing simulations.

The ray tracing has been performed with SYNRAD+ [35,59], a Monte Carlo code which allows coupled vacuum simulations if used along with MOLFLOW+. SYNRAD+ includes a predefined library of reflectivity data.

The results of the power distribution map for the FCC-hh BS in an arc dipole, with $14.069 \mathrm{~m}$ of magnetic length [2] are shown in Fig. 13. The curvature of the proton beam can be noticed, as well as the photon trajectories originating tangentially from it, represented in green. The simulation has been carried out with a nonideal $50 \mathrm{TeV}, 500 \mathrm{~mA}$ beam. $\beta$ has been set to $355 \mathrm{~m}$, the momentum offset $\delta p / p$ to $0.06 \%$ and the normalized emittance $\varepsilon_{N}$ to $2.2 \mu \mathrm{m}$ [2]. For copper and steel a general roughness ratio $\tau=S_{q} / T=$ 0.006 has been assigned, where $S_{q}$ is the RMS surface roughness and $T$ the autocorrelation length. The physical interpretation of $T$ is that it expresses the minimal distance between two profile points not interrelated, giving information about the surface spatial complexity. $\tau$ has been conservatively chosen according to a series of metrology

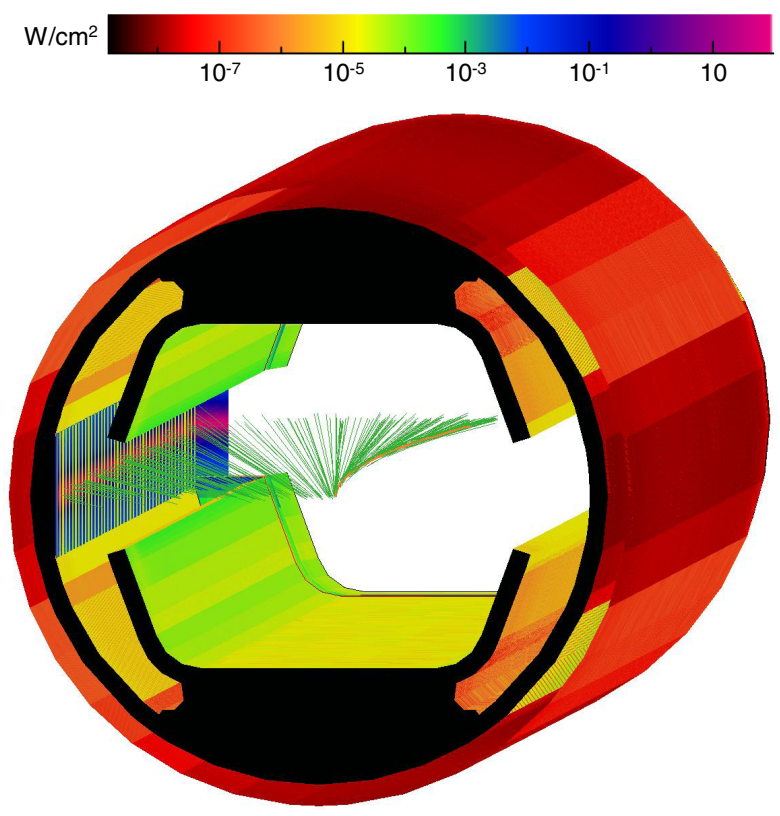

FIG. 13. Ray tracing results of the synchrotron radiation power generated by a $50 \mathrm{TeV}, 500 \mathrm{~mA}$ beam in a standard arc dipole chamber.

studies performed on LHC BS samples at CSEM (Swiss Center for Electronics and Microtechnology). LASE areas have been set as perfectly absorbing surfaces in order to obtain pessimistic results of photoelectron generation and power deposited on the inner chamber.

Looking at the color scale of Fig. 13 and the summary in Table IV it can be noticed how most of the power is absorbed on the first impact region of the SR beam, the sawtooth area on the left baffle. Owing to its high SR absorption, the cold bore and other areas receive a minimum amount of SR power, fulfilling the beam screen's main purpose. The Gaussian-like SR power distribution emitted by the proton beam can be recognized on the sawtooth region, as previously shown in Fig. 5.

The linear power density along the $\mathrm{BS}$ of the $\mathrm{MB}$ is displayed in Fig. 14. The highest value can be found at the beginning of the BS $(\approx 40 \mathrm{~W} / \mathrm{m})$, after the initial region of shadow produced by the SR absorber, and decreases

TABLE IV. SR power distribution per MB.

\begin{tabular}{lcc}
\hline \hline Area & Power & $\%$ of total SR \\
\hline Irradiated baffle & $439 \mathrm{~W}$ & $88 \%$ \\
End absorber & $50.5 \mathrm{~W}$ & $10.1 \%$ \\
Non-irradiated baffle & $6.5 \mathrm{~W}$ & $1.3 \%$ \\
Inner copper primary chamber & $0.6 \mathrm{~W}$ & $0.1 \%$ \\
Interconnect & $0.1 \mathrm{~W}$ & $0.02 \%$ \\
Cold bore & $<0.01 \mathrm{~W}$ & $<0.01 \%$ \\
Other BS areas & $<0.01 \mathrm{~W}$ & $<0.01 \%$ \\
Total per arc dipole & $498.7 \mathrm{~W}$ & $100 \%$ \\
\hline \hline
\end{tabular}




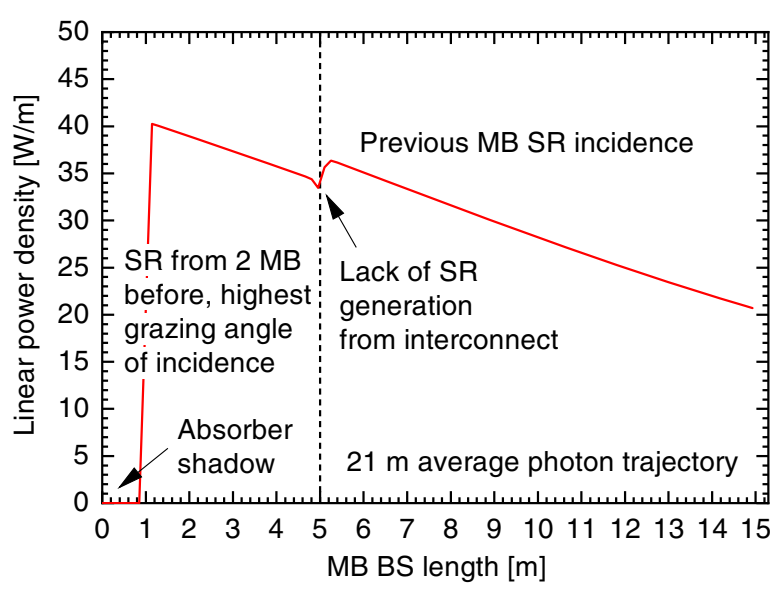

FIG. 14. Linear power density of SR impinging the BS sawtooth surface, for the most irradiated MB, $50 \mathrm{TeV} 500 \mathrm{~mA}$.

progressively along the BS following the beam direction, with the exception of one small jump after $5 \mathrm{~m}$ owing to the change of magnetic region origin. The linear power decay is ascribed to the progressive beam curvature in the previous MB. The beam curvature decreases the SR angle of incidence against the wall, causing a higher spread of the photon fan and lowering its intrinsic power density. The average power received by the sawtooth is around $29 \mathrm{~W} / \mathrm{m}$.

The SR ray tracing allows the determination of the SR energy spectrum arriving to each region. Figure 15 shows an example, representing the spectrum above $1 \mathrm{eV}$ of the SR hitting the horizontal faces of the inner chamber (namely, the areas between which the electron multipacting effect takes place in dipoles) and the SR arriving to the cold bore. It can be seen that most part of the photon flux arriving to these regions carries an energy below copper and SS's work functions, effectively keeping the gas desorption and the $\mathrm{e}^{-}$cloud effect under control.

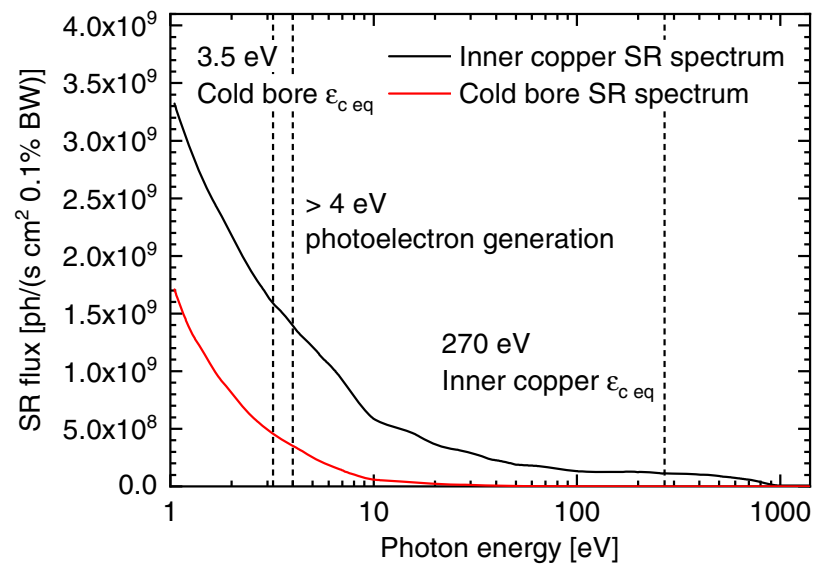

FIG. 15. Spectrum of the synchrotron radiation arriving to the cold mass, after being leaked through the pumping holes, and arriving to the inner chamber, with most part of their flux below copper's work function. $\varepsilon_{c}$ has been calculated without taking into account the part of the spectrum below $1 \mathrm{eV}$.

\section{MECHANICAL ANALYSIS}

The BS has been designed to ensure an elastic behavior after a magnet quench. Eddy currents are induced in the beam screen along its beam axis and, therefore, Lorentz forces squeeze the BS as seen in Fig. 18.

The numerical model used for the magnet quench study is based on the reduced field formulation by means of which no magnet coil needs to be considered. The induced resistive losses affecting the material properties are taken into account. The detailed description of the model can be found in [60].

The formulation of the specific Lorentz forces for a dipole magnetic field is expressed by:

$$
f_{x}=B_{y} \frac{\partial B_{y}}{\partial t} x \sigma(T)
$$

where $f_{x}$ is the volumetric force, $B_{y}$ the magnetic field, $x$ the horizontal distance from the center of the beam screen and $\sigma(T)$ the electrical conductivity as a function of the temperature.

One quarter of the periodic unit $(17 \mathrm{~mm}$ long, see Fig. 18) has been modeled to study the mechanical response of the magnet quench in a time dependent study.

\section{A. Magnet quench behavior}

The evolution of the magnetic field decay [61] and the forces induced in each half of the beam screen is shown in Fig. 16. The forces attain around $135 \mathrm{kgf} / \mathrm{cm}$ on the inner chamber and $44 \mathrm{kgf} / \mathrm{cm}$ on the outer one along the axial direction.

The displacement and the von Mises map of the beam screen when the Lorentz forces are the highest, i.e., at $55 \mathrm{~ms}$, are shown in Fig. 17 and Fig. 18, respectively. The highest stresses are located around the aperture of the secondary chamber. Even if the maximum von Mises stress

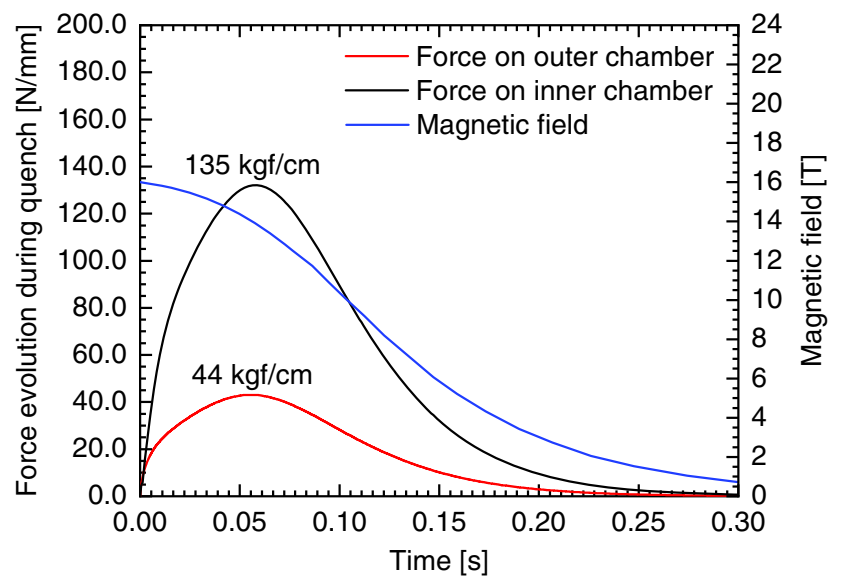

FIG. 16. Comparison of the magnetic field decay during a quench and the resulting force on the beam screen, for a $16 \mathrm{~T}$ dipole. 


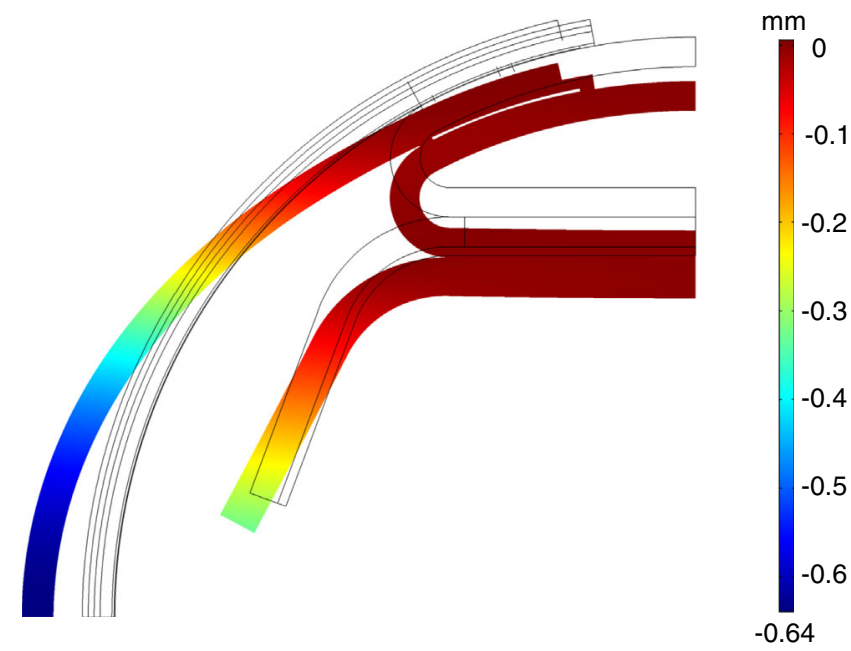

FIG. 17. Maximum displacement of the beam screen in the horizontal direction during a magnet quench.

reaches values as high as $1100 \mathrm{MPa}$, it is very local and it is below the yield strength of the P506 SS, i.e., $1180 \mathrm{MPa}$ at $77 \mathrm{~K}$ [17]. The maximum horizontal displacement during a quench is $0.64 \mathrm{~mm}$, which is less than the gap of $1.5 \mathrm{~mm}$ between the BS and the cold bore.

\section{B. Supporting system}

The supporting system consists of two concentric rings on top of which five elastic fingers $\mathrm{V}$ shaped are welded, see Figs. 19 and 20. Only one ring is welded on the beam screen while the other ring is free to slide and allows, therefore, an adequate insertion and alignment of the BS within the cold bore. The elastic fingers on the horizontal plane have to withstand the expansion of the BS during a magnet quench without any significant plastic deformation.

A radial prestress, due to an imposed radial displacement of $0.1 \mathrm{~mm}$, is applied on each elastic ring to keep the beam screen well positioned with respect to the cold bore. The weight of the beam screen, $2.16 \mathrm{~kg} / \mathrm{m}$, causes a vertical displacement of $-32 \mu \mathrm{m}$. During a quench, the
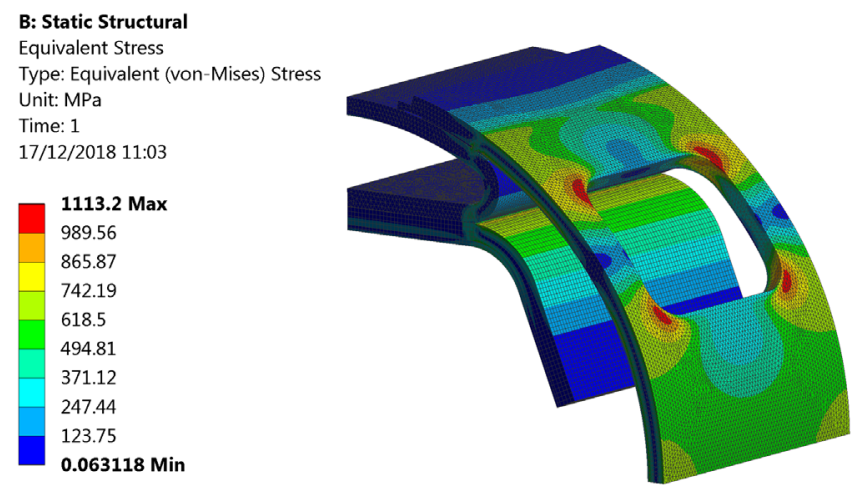

FIG. 18. Highest von Mises stress, in MPa, of the beam screen during a magnet quench.

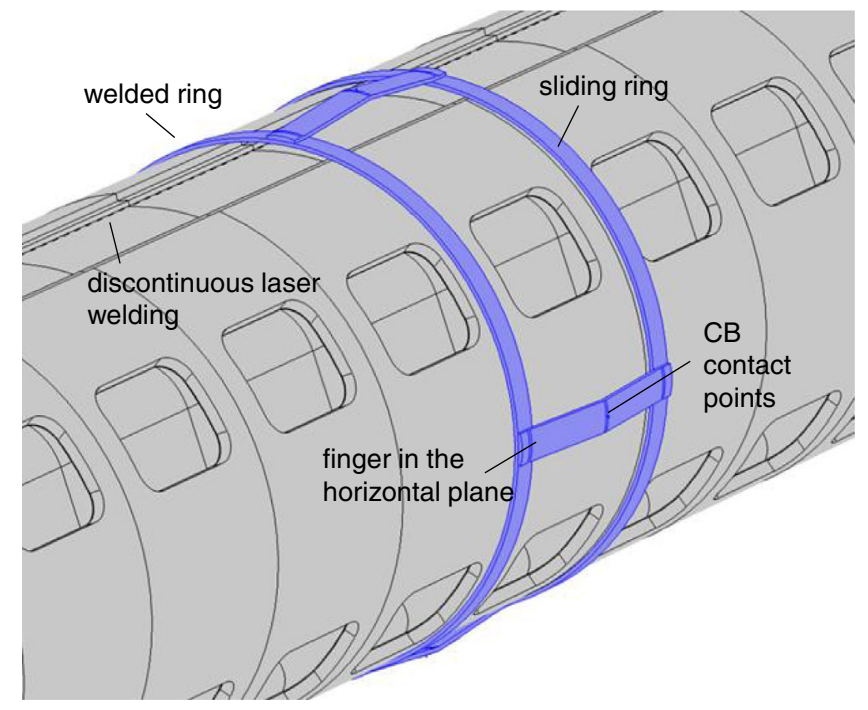

FIG. 19. Supporting system of the FCC-hh BS inside the cold bore, designed to minimize the heat transfer.

most loaded elastic fingers are the ones on the horizontal plane. They are squeezed toward the cold bore by $0.64 \mathrm{~mm}$. After a quench, their residual deformation turns out to be $20 \mu \mathrm{m}$. It is five times lower than the prestress and it is deemed, therefore, negligible. The residual von Mises stress in the horizontal fingers attains values up to around $500 \mathrm{MPa}$, see Fig. 20. However, these values are very localized and not detrimental.

\section{THERMAL ANALYSIS}

The temperature behavior of the beam screen has been simulated by means of the Heat Transfer in Solids and the Heat Transfer with Surface-to-Surface Radiation modules of COMSOL Multiphysics [62].

A specific geometry has been developed for the thermal analysis to take into account the various welds and the thermal contacts between interfacing components. To this purpose, the colaminated copper layers are considered as fully bonded. The welds between the secondary chamber and the cooling channels have been modeled by taking into

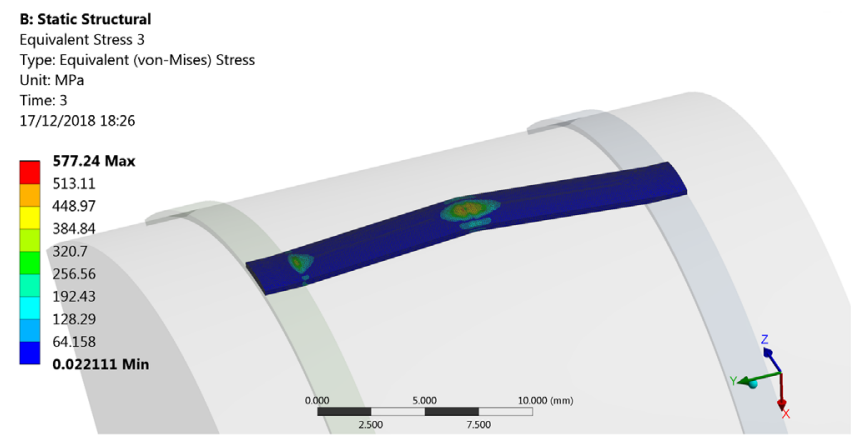

FIG. 20. Residual von Mises stress of the horizontal elastic fingers after a quench. 
account the actual spot welding pattern. To reflect such a pattern, an array of $500 \times 500 \times 75 \mu \mathrm{m}^{3}$ bonding elements placed every $1 \mathrm{~mm}$ has been implemented. The weld between the cooling channel and the inner chamber has been modeled by considering a bonded surface $500 \mu \mathrm{m}$ wide along the external edges of the channel and no contact along the remaining portion.

The contact surface between the rings of the supporting system and the beam screen is considered as fully bonded. The contact area between the elastic finger and the cold bore has been calculated according to the Hertzian theory of nonadhesive elastic contact [63]. Such area turns out to be $0.01 \mathrm{~mm}^{2}$ and it has been used to dimension a cylindrical element $0.1 \mu \mathrm{m}$ high bonding the cold bore to the elastic spring. By adopting this modeling trick, the exact contact area of the thermal transfer is guaranteed. All the thermal contacts have been conservatively considered as fully bonded.

The material properties have been assigned as a function of the temperature. The heat capacity at constant pressure for the P506 is taken from [64] and for the copper from [65], while the thermal conductivity for the P506 SS from [66] and for the copper from [67]. All the internal surfaces involved in the thermal radiation have been considered as gray surfaces, while an insulation condition has been applied on the external surfaces of the cold bore. The surface emissivity of copper in the BS, considered to be at $77 \mathrm{~K}$, has been set to 0.12 and for the P506 to 0.34. For the $1.9 \mathrm{~K}$ cold bore the emissivity is set to 0.12 [68].

To simplify the meshing of the beam screen, the area of impact of the SR has been divided in seven longitudinal regions of equal area, vertically aligned, and an absolute heat load has been applied to each one of them, emulating the typical Gaussian distribution of the SR radiation (see Fig. 5) as a 7-step function.

The temperature map of a short model has been compared with the exact Gaussian profile of the heat load resulting in a good match, since the high thermal conductivity of copper and given that the total heat load of the simplified model is the same.

Each periodic portion of the beam screen has been discretized with 395, 479 tetrahedral elements resulting in an average element quality of 0.57 . The analysis has been performed in stationary conditions for the lowest and highest temperature of the coolant, $40 \mathrm{~K}$ and $57 \mathrm{~K}$.

\section{A. Temperature of the beam screen in nominal conditions}

The modeling of a periodic unit of the beam screen (17 mm long) is sufficient to determine the temperature distribution in nominal conditions. The main source of heat is the SR, around $40 \mathrm{~W} / \mathrm{m}$ at the highest point, as represented in Fig. 14. Other minor loads are the image currents and the $\mathrm{e}^{-}$cloud, with a budget of around $3 \mathrm{~W} / \mathrm{m}$ [4] and $0.1 \mathrm{~W} / \mathrm{m}$, respectively. The heat load intercepted in

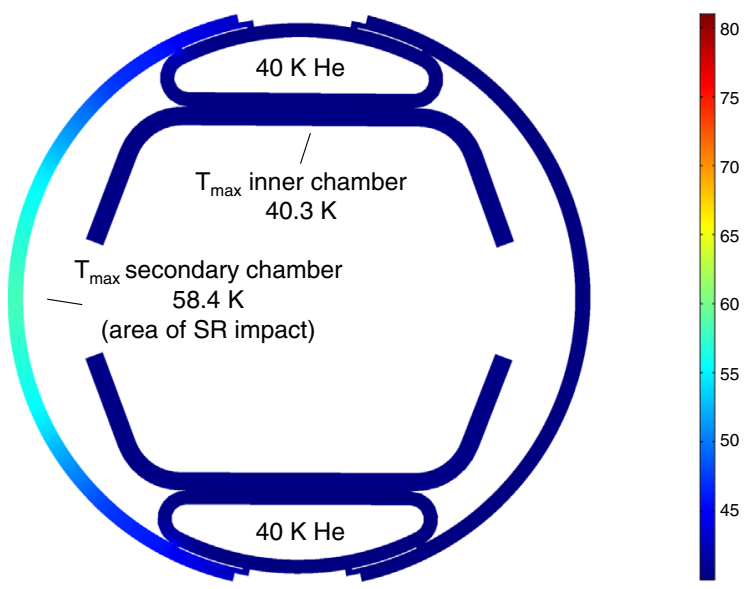

FIG. 21. Temperature distribution in the BS cross section at the most irradiated length. He at $40 \mathrm{~K}$, with a $50 \mathrm{TeV}, 500 \mathrm{~mA}$ beam.

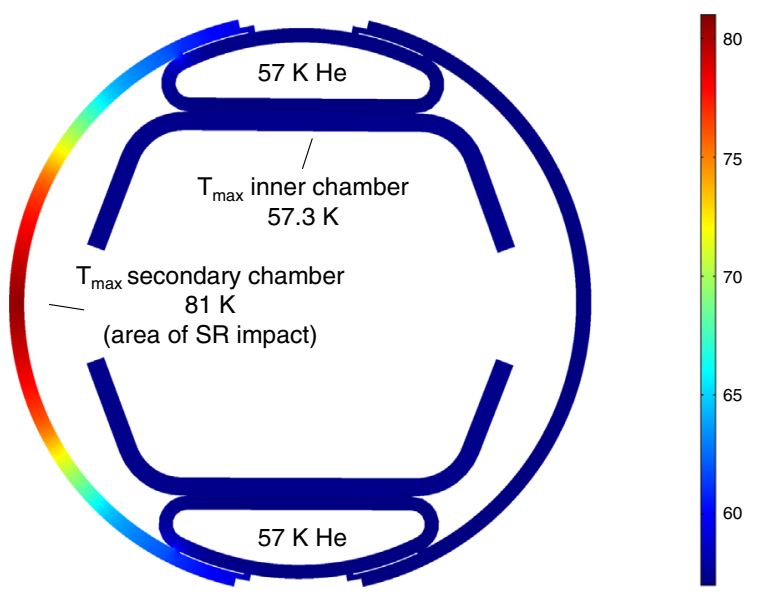

FIG. 22. Temperature distribution in the BS cross section at the most irradiated length. He at $57 \mathrm{~K}$, with a $50 \mathrm{TeV}, 500 \mathrm{~mA}$ beam.

the secondary chamber is transferred through the copper layer and, ultimately, through the welding points joining the secondary chamber to the cooling channels. The heat transfer is limited by the P506 SS as in this temperature range, its thermal conductivity is around a factor 100 lower than copper. The temperature map of the BS, considering the maximum SR power load and the He inlet temperature of $40 \mathrm{~K}$ and $57 \mathrm{~K}$, is shown in Figs. 21 and 22, respectively.

As the inner chamber is thermally decoupled from the secondary one where the SR is intercepted, the temperature of the inner chamber increases by $0.3 \mathrm{~K}$ above the base temperature of the cooling channel. Therefore, such temperature remains within the defined range between $40 \mathrm{~K}$ and $60 \mathrm{~K}$ needed to maintain the beam impedance low.

\section{B. Expected heat loads on the $1.9 \mathrm{~K}$ cold mass}

For each magnet the $40 \%$ of its thermal budget is represented by the heat loads from inside the cold bore, i.e., $0.3 \mathrm{~W} / \mathrm{m} /$ aperture [6]. 


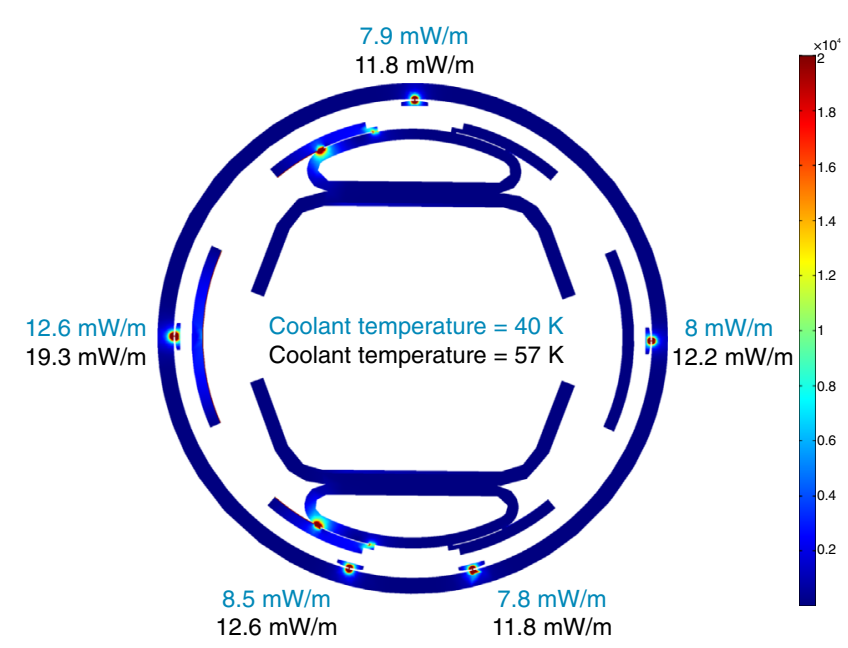

FIG. 23. Heat losses to the cold bore through each elastic finger. The heat loss $[\mathrm{mW} / \mathrm{m}]$ is represented in blue and in black for a cooling temperature of $40 \mathrm{~K}$ and $57 \mathrm{~K}$, respectively. Color scale in arbitrary units.

The nuclear scattering accounts for most of the total heat load. Its average contribution has been calculated with Eq. (2).

A beam screen assembly $750 \mathrm{~mm}$ long has been modeled to determine the heat losses to the $1.9 \mathrm{~K}$ cold bore from the supporting system as it is placed every $750 \mathrm{~mm}$. Its maximum heat loss is estimated to be around $45 \mathrm{~mW} / \mathrm{m}$ for a base temperature of $40 \mathrm{~K}$ and $67.7 \mathrm{~mW} / \mathrm{m}$ for a base temperature of $57 \mathrm{~K}$, see Fig. 23.

The other considered heat sources are the thermal radiation produced by the $40-60 \mathrm{~K} \mathrm{BS}$ and the leaked $\mathrm{SR}$, both of them playing a minor role. All the heat sources are displayed in Table V along with their ratio over the total.

In average, the total heat load is situated well within the budget. Nevertheless, it is expected to be surpassed at some points owing to the high variability of the nuclear scattering power deposition along the cell elements. Considering only this source, the cold mass of the most impacted dipole can receive up to $278 \mathrm{~mW} / \mathrm{m} \mathrm{[10].}$

TABLE V. Estimated heat loads on the cold mass per beam aperture in the hotter part of the BS, for baseline parameters. Nuclear scattering calculated as an average along the arc cell.

\begin{tabular}{lcc}
\hline \hline Heat source & Value & Percentage \\
\hline Nuclear scattering & $178 \mathrm{~mW} / \mathrm{m}$ & $69.8 \%$ \\
Conduction through BS supports & $67.7 \mathrm{~mW} / \mathrm{m}$ & $26.5 \%$ \\
BS thermal radiation & $8.9 \mathrm{~mW} / \mathrm{m}$ & $3.5 \%$ \\
Leaked SR power & $0.5 \mathrm{~mW} / \mathrm{m}$ & $0.2 \%$ \\
Total & $255.1 \mathrm{~mW} / \mathrm{m}$ & $100 \%$ \\
\hline \hline
\end{tabular}

\section{CONCLUSIONS}

A new beam vacuum chamber design for the FCC-hh has been presented. It is intended to overcome the challenges derived from the increase of the state-of-art beam energy, from the $7 \mathrm{TeV}$ of the LHC up to the $50 \mathrm{TeV}$ of the FCC-hh, which raises the linear power density from $0.22 \mathrm{~W} / \mathrm{m}$ up to $35.4 \mathrm{~W} / \mathrm{m}$. The design aims to minimize the electron cloud build up, the outgassing triggered by the synchrotron radiation and the heat leakage to the cold mass, maximizing at the same time the beam screen's pumping efficiency. The performed calculations have shown a pumping speed more than three times higher than the LHC's, a heat transfer to the cold mass within the heat budget, and an $\mathrm{e}^{-}$cloud density below the instability limits. The $\mathrm{e}^{-}$cloud would be effectively suppressed thanks to the new SEY mitigation features, not present in the LHC, and the low reflectivity properties of the sawtooth finishing. In spite of having a SR linear power density around 160 times higher than the LHC, the FCC-hh beam screen is able to keep cold the copper surfaces surrounding the beam, keeping the resistivity low. All the stresses generated during magnet quenches are also well sustained. The resulting design complexity is much higher than that of the LHC's, but still economically affordable in a large scale production. As open points remain the precise calculation of LASE's impact on the impedance and the determination of its exact manufacturing features match in an optimal way the collider requirements.

\section{ACKNOWLEDGMENTS}

The authors would like to express their gratitude to R. Cimino and the LNF-INFN team for the collaboration arranged to study the reflectivity and photoelectron yield of the beam screen materials. The authors would also like to thank the EuroCirCol WP4 collaboration for the interesting discussions, M. Ady for his support on the photon ray tracing and J. R. Hunt and A. Infantino for their detailed calculations on proton scattering. This work was supported by the European Union's Horizon 2020 research and innovation programme under Grant No. 654305.

[1] M. Benedikt, European Circular Energy Frontier Collider Study, H2020-INFRADEV-1-2014-1, Report No. 654305, 2014.

[2] M. Benedikt, M. C. Garrido, F. Cerutti, B. Goddard, J. Gutleber, J. M. Jimenez, M. Mangano, V. Mertens, J. A. Osborne, T. Otto, J. Poole, W. Riegler, D. Schulte, L. J. Tavian, D. Tommasini, and F. Zimmermann, Future Circular Collider, Tech. Report No. CERN-ACC-20180058 (CERN, Geneva, 2018) Volume 3-The Hadron Collider (FCC-hh).

[3] O. Gröbner, Overview of the LHC vacuum system, Vacuum 60, 25 (2001). 
[4] P. Lebrun and L. Tavian, Beyond the large hadron collider: A first look at cryogenics for CERN future circular colliders, Phys. Procedia 67, 768 (2015).

[5] I. Bellafont, L. Mether, R. Kersevan, O. Malyshev, V. Baglin, P. Chiggiato, and F. Pérez, Beam induced vacuum effects in the future circular hadron collider beam vacuum chamber, Phys. Rev. Accel. Beams (to be published).

[6] C. Kotnig, Cold mass cooling with supercritical helium, 2nd FCC Cryogenics Day (2016).

[7] O. S. Bruning, P. Collier, P. Lebrun, S. Myers, R. Ostojic, J. Poole, and P. Proudlock, LHC Design Report Vol.1: The LHC Main Ring (CERN, Geneva, Switzerland, 2004), Chap. 12.

[8] T. Böhlen, F. Cerutti, M. Chin, A. Fasso, A. Ferrari, P. Ortega, A. Mairani, P. Sala, G. Smirnov, and V. Vlachoudis, The FLUKA code: Developments and challenges for high energy and medical applications, Nucl. Data Sheets 120, 211 (2014).

[9] A. Ferrari, P. R. Sala, A. Fasso, and J. Ranft, FLUKA: A multi-particle transport code, https://doi.org/10.2172/ 877507.

[10] J. R. Hunt, Update on R2E and heat load simulations, FCC Week (2019), https://indico.cern.ch/event/727555/ contributions/3449897/.

[11] J. R. Hunt (private communication).

[12] V. Baglin, G. Bregliozzi, J. M. Jimenez, and G. Lanza, in Proceedings of the 2nd International Particle Accelerator Conference, San Sebastián, Spain (EPS-AG, Spain, 2011), Vol. C110904, pp. 1563-1565.

[13] A. Hofmann, in CERN Accelerator School (CAS) 1989 (1989), https://cds.cern.ch/record/202177/files/cer-000113842 .pdf.

[14] O. Gröbner, Dynamic outgassing, Technical Report No. CERN-OPEN-2000-275, 1999.

[15] V. Baglin, P. Lebrun, L. Tavian, and R. van Weelderen, Cryogenic beam screens for high-energy particle accelerators, CERN Technical Report No. CERN-ATS-2013-006, 2013.

[16] V. V. Anashin, G. Derevyankin, V. G. Dudnikov, O. B. Malyshev, V. N. Osipov, C. L. Foerster, F. M. Jacobsen, M. W. Ruckman, M. Strongin, R. Kersevan, I. L. Maslennikov, W. C. Turner, and W. A. Lanford, Cold beam tube photodesorption and related experiments for the Superconducting Super Collider Laboratory $20 \mathrm{TeV}$ proton collider, J. Vacuum Sci. Technol. A 12, 1663 (1994).

[17] S. Sgobba and G. Hochortler, A new non-magnetic stainless steel for very low temperature applications, in Proceedings of the International Congress Stainless Steel 1999: Science and Market, Chia Laguna, Italy, Vol. 2 (Associazione Italiana di Metallurgia, Milano, 1999), pp. 391-401.

[18] E. Metral, Beam screen issues, CERN Yellow Report CERN-2011-003, 2011, pp. 83-89.

[19] A. Sven, Betatron collimation system insertion, FCC Week (2017), https://indico.cern.ch/event/556692/contributions/ 2484254/.

[20] P. Duthil, in Proceedings of the CAS - CERN Accelerator School: Course on Superconductivity for Accelerators, Erice (2014), pp. 77-95, https://doi.org/10.5170/CERN2014-005.77.
[21] A. Sven (private communication).

[22] D. Astapovych (private communication).

[23] R. Valizadeh, O. B. Malyshev, S. Wang, S. A. Zolotovskaya, W. A. Gillespie, and A. Abdolvand, Low secondary electron yield engineered surface for electron cloud mitigation, Appl. Phys. Lett. 105, 231605 (2014).

[24] R. Valizadeh, O. Malyshev, S. Wang, T. Sian, M. Cropper, and N. Sykes, Reduction of secondary electron yield for E-cloud mitigation by laser ablation surface engineering, Appl. Surf. Sci. 404, 370 (2017).

[25] R. Valizadeh, M. Cropper, P. Goudket, O. Malyshev, B. Sian, N. Sykes, and S. Wang, in Proceedings of IPAC2016, Busan, Korea, 2016 (2016) p. 089-1092, http://inspirehep .net/record/1469811/files/tuocb02.pdf.

[26] G. Tang, A. C. Hourd, and A. Abdolvand, Nanosecond pulsed laser blackening of copper, Appl. Phys. Lett. 101, 231902 (2012).

[27] P. C. Pinto, S. Calatroni, H. Neupert, D. Letant-Delrieux, P. Edwards, P. Chiggiato, M. Taborelli, W. Vollenberg, C. Yin-Vallgren, J. Colaux, and S. Lucas, Carbon coatings with low secondary electron yield, Vacuum 98, 29 (2013).

[28] C. Y. Vallgren, G. Arduini, J. Bauche, S. Calatroni, P. Chiggiato, K. Cornelis, P. Pinto, B. Henrist, E. Metral, H. Neupert, G. Rumolo, E. Shaposhnikova, and M. Taborelli, Amorphous carbon coatings for the mitigation of electron cloud in the CERN Super Proton Synchrotron, Phys. Rev. Accel. Beams 14, 071001 (2011).

[29] R. Valizadeh, A. Hannah, J. Much, D. Whitehead, P. Krkotic, O. Malyshev, J. M. O'Callaghan, and M. Pont, Evaluation of LASER ablated surface engineering of copper and stainless steel for particle accelerators, FCC Week (2019), https://indico.cern.ch/event/727555/ contributions/3468921.

[30] S. Calatroni, M. Arzeo, S. Aull, M. Himmerlich, P. Costa Pinto, W. Vollenberg, B. Di Girolamo, P. Cruikshank, P. Chiggiato, D. Bajek, S. Wackerow, and A. Abdolvand, Cryogenic surface resistance of copper: Investigation of the impact of surface treatments for secondary electron yield reduction, Phys. Rev. Accel. Beams 22, 063101 (2019).

[31] A. Sokolov, F. Eggenstein, A. Erko, R. Follath, S. Künstner, M. Mast, J. Schmidt, F. Senf, F. Siewert, T. Zeschke et al., in Advances in Metrology for X-Ray and EUV Optics $V$ (International Society for Optics and Photonics, Lausanne, Switzerland, 2014), Vol. 9206, p. 92060J.

[32] E. La Francesca, M. Angelucci, A. Liedl, L. Spallino, L. A. Gonzalez, I. Bellafont, F. Siewert, M. G. Sertsu, A. Sokolov, F. Schäfers, and R. Cimino, Photo reflectivity and photoelectron yield from copper technical surfaces (to be published).

[33] E. La Francesca, A. Liedl, M. Angelucci, A. Sokolov, M. G. Sertsu, F. Schäfers, F. Siewert, and R. Cimino, Study of Reflectivity and Photo Yield on FCC-hh proposed beam screen surfaces, FCC Week (2018), https://indico.cern.ch/ event/656491/contributions/2938727/.

[34] A. Liedl, E. La Francesca, M. Angelucci, and R. Cimino, Photo reflectivity and photo electron yield of technical surfaces, e-Cloud Workshop, Isola d'Elba, Italy (2018), https://agenda.infn.it/event/13351/contributions/18931/. 
[35] M. Ady and R. Kersevan, MOLFLOW+ and SYNRAD+ Website (2018), https://molflow.web.cern.ch/.

[36] L. A. Gonzalez, V. Baglin, I. Bellafont, S. Casalbuoni, P. Chiggiato, C. Garion, E. Huttel, R. Kersevan, and F. Pérez, Photodesorption Studies on FCC-hh Beam Screen Prototypes at KARA, FCC Week (2019), https://indico.cern.ch/ event/727555/contributions/3447250/.

[37] A. Romano, G. Iadarola, K. Li, and G. Rumolo, in Proceedings of IPAC 2016 (2016), pp. 1454-1457, http://jacow.org/ipac2016/papers/tupmw016.pdf.

[38] S. Arsenyev and D. Schulte, in Proceedings of IPAC 2018 (2018), pp. 153-156, http://accelconf.web.cern.ch/ AccelConf/ipac2018/doi/JACoW-IPAC2018-MOPMF030 .html.

[39] M. Chorowski, H. Correia, D. Delikaris, P. Duda, C. Haberstroh, F. Holdener, S. Klöppel, C. Kotnig, F. Millet, J. Polinski, H. Quack, and L. Tavian, in Cryogenic Engineering Conference and International Cryogenic Materials Conference 2017 (CEC/ICMC'17), Madison, Wisconsin (USA), Vol. 278 (IOP Publishing, 2017), p. 012097.

[40] C. Kotnig (private communication).

[41] N. Kos, LHC Beam screen insertion tests with sliding rings, CERN Technical Report No. VACUUM-TECHNICAL-NOTE-00-06, 2000.

[42] L. R. Evans, The Large Hadron Collider: A Marvel of Technology (EPFL Press, 2009), pp. 91-92.

[43] C. Garion, Considerations for large scale production of the FCC-hh beam screens, FCC Week (2019), https://indico .cern.ch/event/727555/contributions/3476022/.

[44] M. Benedikt et al. (private communication).

[45] M. Ady and R. Kersevan, in Proceedings of IPAC 2014, Dresden, Germany (JACoW, 2014), pp. 2348-2350, http://accelconf.web.cern.ch/AccelConf/IPAC2014/papers/ wepme038.pdf.

[46] M. Ady, Monte Carlo simulations of ultra high vacuum and synchrotron radiation for particle accelerators, Ph.D. thesis, Ecole Polytechnique, Lausanne, 2016.

[47] G. Iadarola and G. Rumolo, in Proceedings of the Joint INFNCERN-EuCARD-AccNet Workshop on Electron-Cloud Effects, La Biodola, Isola d'Elba, Italy, 2012 (2013), pp. 189194, https://doi.org/10.5170/CERN-2013-002.189.

[48] G. Iadarola, G. Rumolo, and G. Miano, Electron cloud studies for CERN particle accelerators and simulation code development, Ph.D. thesis, CERN, 2014, https://cds.cern .ch/record/1705520.

[49] M. A. Furman and M. T. F. Pivi, Probabilistic model for the simulation of secondary electron emission, Phys. Rev. Accel. Beams 5, 124404 (2002).

[50] R. E. Kirby and F. K. King, Secondary electron emission yields from PEP-II accelerator materials, Nucl. Instrum. Methods Phys. Res., Sect. A 469, 1 (2001).

[51] R. Cimino, I. R. Collins, M. A. Furman, M. Pivi, F. Ruggiero, G. Rumolo, and F. Zimmermann, Can LowEnergy Electrons Affect High-Energy Physics Accelerators?, Phys. Rev. Lett. 93, 014801 (2004).

[52] V. Baglin, I. Collins, B. Henrist, N. Hilleret, and G. Vorlaufer, A summary of main experimental results con- cerning the secondary electron emission of copper, Tech. Report No. LHC-Project-Report-472 (CERN, Geneva, 2001).

[53] B. Henrist, N. Hilleret, M. Jimenez, C. Scheuerlein, M. Taborelli, and G. Vorlaufer, in Proceedings of ECLOUD'02: Mini-Workshop on Electron-Cloud Simulations for Proton and Positron Beams, CERN, Geneva, Switzerland, 2001 (2002), p. 309, http://cern.ch/conf-ecloud02/papers/allpdf/ hilleret.pdf.

[54] R. Cimino and I. Collins, Vacuum chamber surface electronic properties influencing electron cloud phenomena, Appl. Surf. Sci. 235, 231 (2004).

[55] F. Pérez, P. Chiggiato, O. Malyshev, R. Valizadeh, T. Sian, and R. Sirvinskaite, Proposal on surface engineering to mitigate electron cloud effects, Technical Report No. EuroCirCol-P2-WP4-M4.4, 2017.

[56] K. Ohmi, F. Zimmermann, and E. Perevedentsev, Wakefield and fast head-tail instability caused by an electron cloud, Phys. Rev. E 65, 016502 (2001).

[57] K. Ohmi, in Proceedings of ECLOUD'04 (CERN, Geneva, 2005), https://cds.cern.ch/record/847899.

[58] S. Calatroni, E. Garcia-Tabares, H. Neupert, V. Nistor, A. T. Pérez, M. Taborelli, P. Chiggiato, O. B. Malyshev, R. Valizadeh, S. Wackerow, S. A. Zolotovskaya, W. A. Gillespie, and A. Abdolvand, First accelerator test of vacuum components with laser-engineered surfaces for electron-cloud mitigation, Phys. Rev. Accel. Beams 20, 113201 (2017).

[59] R. Kersevan, in Proceedings of International Conference on Particle Accelerators, Vol. C930517 (IEEE, 1993), pp. 3848-3850.

[60] M. Morrone, C. Garion, M. Aurisicchio, and P. Chiggiato, A coupled multiphysics FEM model to investigate electromagnetic, thermal and mechanical effects in complex assemblies: The design of the High-Luminosity Large Hadron Collider beam screen, Appl. Math. Model. 57, 280 (2018).

[61] D. Schoerling (private communication).

[62] COMSOL Multiphysics Reference Manual (2017).

[63] R. G. Budynas, J. K. Nisbett et al., Shigley's Mechanical Engineering Design (McGraw-Hill, New York, 2008), Vol. 8.

[64] J. Corsan and N. Mitchem, in Proc. 6th Int. Cryogenic Eng. Conf., 1976, edited by K. Mendelssohn (IPC Science and Technology Press, London, 1976), p. 527.

[65] G. K. White and S. Collocott, Heat Capacity of Reference Materials: $\mathrm{Cu}$ and W, J. Phys. Chem. Ref. Data 13, 1251 (1984).

[66] S. Sgobba (private communication).

[67] E. Drexler, N. Simon, and R. Reed, Properties of copper and copper alloys at cryogenic temperatures, NIST Technical Report Nos. PB-92-172766/XAB; NIST/MONO177, 1992.

[68] R. F. Barron et al., Cryogenic Heat Transfer, Chemical and Mechanical Engineering (Taylor and Francis, Philadelphia, PA, 1999), https://cds.cern.ch/record/582975. 